\title{
Pulsed electromagnetic fields potentiate the paracrine function of mesenchymal stem cells for cartilage regeneration
}

\author{
Dinesh Parate ${ }^{1,2,3}$, Nurul Dinah Kadir ${ }^{3}$, Cenk Celik ${ }^{3}$, Eng Hin Lee ${ }^{3,4}$, James H. P. Hui ${ }^{3,4}$, \\ Alfredo Franco-Obregón ${ }^{1,2,5^{*}}$ and Zheng Yang ${ }^{3,4^{*}}$ (i)
}

\begin{abstract}
Background: The mesenchymal stem cell (MSC) secretome, via the combined actions of its plethora of biologically active factors, is capable of orchestrating the regenerative responses of numerous tissues by both eliciting and amplifying biological responses within recipient cells. MSCs are "environmentally responsive" to local micro-environmental cues and biophysical perturbations, influencing their differentiation as well as secretion of bioactive factors. We have previously shown that exposures of MSCs to pulsed electromagnetic fields (PEMFs) enhanced MSC chondrogenesis. Here, we investigate the influence of PEMF exposure over the paracrine activity of MSCs and its significance to cartilage regeneration.
\end{abstract}

Methods: Conditioned medium (CM) was generated from MSCs subjected to either 3D or 2D culturing platforms, with or without PEMF exposure. The paracrine effects of CM over chondrocytes and MSC chondrogenesis, migration and proliferation, as well as the inflammatory status and induced apoptosis in chondrocytes and MSCs was assessed.

Results: We show that benefits of magnetic field stimulation over MSC-derived chondrogenesis can be partly ascribed to its ability to modulate the MSC secretome. MSCs cultured on either 2D or 3D platforms displayed distinct magnetic sensitivities, whereby MSCs grown in 2D or 3D platforms responded most favorably to PEMF exposure at $2 \mathrm{mT}$ and $3 \mathrm{mT}$ amplitudes, respectively. Ten minutes of PEMF exposure was sufficient to substantially augment the chondrogenic potential of MSC-derived CM generated from either platform. Furthermore, PEMF-induced CM was capable of enhancing the migration of chondrocytes and MSCs as well as mitigating cellular inflammation and apoptosis.

Conclusions: The findings reported here demonstrate that PEMF stimulation is capable of modulating the paracrine function of MSCs for the enhancement and re-establishment of cartilage regeneration in states of cellular stress. The PEMF-induced modulation of the MSC-derived paracrine function for directed biological responses in recipient cells or tissues has broad clinical and practical ramifications with high translational value across numerous clinical applications.

Keywords: Pulse electromagnetic fields, Mesenchymal stem cells, Cartilage, Paracrine

\section{Background}

Articular cartilage is an avascular tissue with limited intrinsic capacity for regeneration. In combination with the limitations of existing treatment modalities, joint injuries often deteriorate with time into articular joint disease $[1,2]$.

\footnotetext{
* Correspondence: suraf@nus.edu.sg; dosyz@nus.edu.sg

'Department of Surgery, National University of Singapore, Singapore 119228, Singapore

${ }^{3}$ Department of Orthopaedic Surgery, Yong Loo Lin School of Medicine, National University of Singapore, NUHS Tower Block, Level 11, 1E Kent Ridge Road, Singapore 119288, Singapore

Full list of author information is available at the end of the article
}

Mesenchymal stem cells (MSCs), with their capacity for expansion and demonstrated multipotency for a variety of tissue lineages, have been championed as a promising cell source for the repair and regeneration of many degenerative, inflammatory, or autoimmune diseases [3]. Nevertheless, despite their anticipated, but at times not substantiated, potential, MSC-based strategies have often fallen short of initial expectations [4]. Although the potential applicability of MSCs for cartilage repair was initially postulated based on their ability to differentiate into chondrocytes and to participate in the formation of tissue, it has

(c) The Author(s). 2020 Open Access This article is distributed under the terms of the Creative Commons Attribution 4.0 International License (http://creativecommons.org/licenses/by/4.0/), which permits unrestricted use, distribution, and 
become increasingly evident that part of the reparative value of MSCs is attributed to their paracrine manner of developmental entrainment [5]. In non-contact co-culture experiments between MSCs and primary chondrocytes, improvements in chondrocyte proliferation, phenotype maintenance, and enhanced matrix synthesis were shown to be credited to MSC-derived trophic factors [6, 7]. A paracrine role of MSCs was further supported by preclinical studies showing that intra-articular injection of MSCs prevented the development of post-traumatic arthritis and promoted cartilage regeneration in damaged joints [8], as well as improved clinical outcomes and indices of cartilage repair in second-look arthroscopy [9].

The spectrum of trophic factors released by MSCs is collectively referred to as the secretome. Via the combined actions of its plethora of biologically active factors, the MSC secretome is capable of establishing a regenerative microenvironment for the repair of injured tissues by both eliciting and amplifying biological responses within recipient cells. Compositional analyses of the secretome using mass spectrometry, next-generation sequencing, or lipid profiling have identified cytokines and growth factors with diverse biological properties that have been implicated in a vast array of cellular processes ranging from cell activation and proliferation to differentiation. Components of the secretome have also been ascribed key roles in critical aspects of tissue regeneration such as promoting angiogenesis, inhibiting apoptosis, immunomodulation, anti-inflammation, and stem cell homing to sites of injury [10-12]. In particular, MSC exosomes were demonstrated to possess therapeutic potential for cartilage repair in osteochondral defects [13, $14]$ as well as protection against cartilage and bone degradation in in vivo models of osteoarthritis $[15,16]$.

The function of the MSC secretome is activated by local micro-environmental cues that modulate MSC differentiation as well as that of subsidiary tissues $[17,18]$. Changes in growth factors/cytokines [19, 20], oxygen tension [21, 22], or environmental mechanical cues arising from the extracellular matrix [23, 24] or substrate stiffness $[25,26]$ have been shown to directly influence MSC paracrine activity. Scaffolds of distinct composition and cytoarchitecture influence how the cellular mechanotransduction machinery translates environmental mechanical and chemical cues into transcriptional and paracrine responses. Moreover, growing MSCs as 3D spheroids encourages cell-cell interactions that upregulate paracrine activities with anti-inflammatory and angiogenic properties [27-29]. Furthermore, diverse forms of mechanical stimulation such as shear stress, tensile stress, and compression have been shown to promote the production of reactive oxygen species (ROS) [30], as well as alter the secretome profile of MSCs [31, 32]. Accordingly, activation of mitochondrial respiration is known to activate the muscular secretome [33].
We have previously shown that pulsed electromagnetic fields (PEMFs) activate calcium-permeable transient receptor potential (TRP) channels, promoting both in vitro chondrogenesis [34] and myogenesis [35] by activating a calcium-mitochondrial transcriptional and epigenetic axes governing survival and development [35]. PEMFinduced MSC differentiation has been correlated with increased expression of TGF $\beta$ and BMP2 [36-38]. Moreover, the expression and paracrine action of these growth factors was also demonstrated in electrically driven MSC chondrogenesis [39]. PEMF exposure has also been shown to exert anti-inflammatory effects by upregulating A2A and A3ARs, thereby mitigating the expression of pro-inflammatory cytokines $[40,41]$. This is supported by studies showing PEMF inhibition of the PGE2 and cycloxigenase-2 (COX-2) pathways, reducing the expression of pro-inflammatory cytokines (IL-6, IL8) while augmenting anti-inflammatory factors (cAMP, IL-10) in synovial fibroblasts from bovine and osteoarthritic patients [42-44]. Here, we provide evidence that the previously described pro-chondrogenic of PEMF stimulation [34] can be largely attributed to its modulation of the MSC secretome (Fig. 1). PEMF stimulation was shown to modulate the chondrogenic, chemotactic, anti-inflammatory, and antiapoptotic activities of the MSC secretome in the form of conditioned medium (CM) harvested from PEMF-exposed MSCs. PEMF stimulation, via its effects over MSC paracrine signaling, might hence represent a manner of promoting regeneration in an inflammatory joint environment.

\section{Methods \\ PEMF exposure system}

The PEMF device used in this study has been previously described [34, 35]. Briefly, the device produces spatially homogeneous, time-varying magnetic fields, consisting of barrages of $20 \times 150 \mu \mathrm{s}$ on and off pulses for $6 \mathrm{~ms}$ repeated at a frequency of $15 \mathrm{~Hz}$. The magnetic flux density rose to predetermined maximal level within $\sim 50 \mu \mathrm{s}$ $(\sim 17 \mathrm{~T} / \mathrm{s})$ when driving field amplitudes between 0.5 and $4 \mathrm{mT}$. Unless explicitly noted, all samples were exposed once for $10 \mathrm{~min}$. All PEMF-treated samples were compared to time-matched control samples $(0 \mathrm{mT})$ that were manipulated in exactly the same manner as experimental samples, including placement into the PEMFgenerating apparatus for the designated time, except that the apparatus was not set to generate a magnetic field.

\section{Human bone marrow MSC culture}

Primary human mesenchymal stem cells (MSCs) were purchased from RoosterBio Inc. (Frederick, MD), supplied at passage 3. MSCs were further expanded in MSC High Performance Media (RoosterBio Inc.) at $37^{\circ} \mathrm{C}$ in 


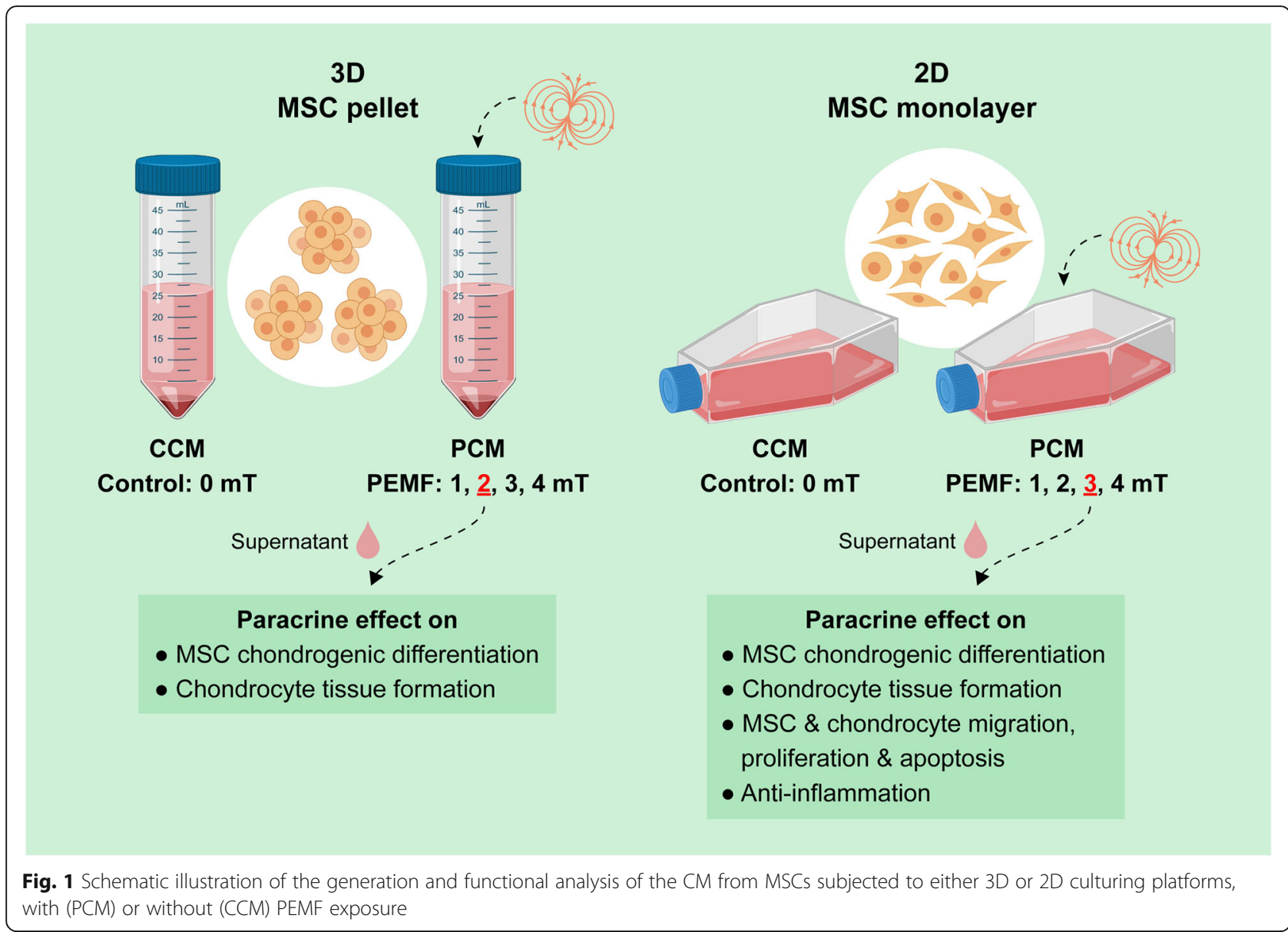

$5 \% \mathrm{CO}_{2}$ atmosphere. The expanded MSCs were used between passages 5 and 6 .

\section{Conditioned media (CM) from MSC in 3D and 2D culture platforms}

Conditioned media $(\mathrm{CM})$ was generated from 3D culture pellets as previously described [34]. Briefly, MSCs were expanded in expansion media until $70-80 \%$ confluency before being subjected to pellet formation $\left(2.5 \times 10^{5}\right.$ cells per pellet) and left in expansion media overnight. The following day the expansion media was replaced with $0.5 \mathrm{ml}$ of low-glucose DMEM (Life Technologies) media without FBS. PEMF treatment was applied for a duration of $10 \mathrm{~min}$ at $1,2,3$, and $4 \mathrm{mT}$, or $2 \mathrm{mT}$ for $30 \mathrm{~min}$. CMs from pellet culture were collected $24 \mathrm{~h}$ post-PEMF exposure and pooled for later use.

To generate CM from 2D culture platforms, MSCs were cultured in T75 flasks at a seeding density of $1.5-2 \times 10^{5}$ in expansion media. At 50-60\% confluency, the expansion media was replaced with $10 \mathrm{ml}$ of low-glucose DMEM media without FBS. PEMF exposure was applied at 1, 2, 3, and $4 \mathrm{mT}$ for $10 \mathrm{~min}$, or $2 \mathrm{mT}$ for $30 \mathrm{~min}$, and the $\mathrm{CM}$ generated (PCM) was collected at $24 \mathrm{~h}$ post-PEMF exposure, pooled, and used for further application and analysis.
Control CM (CCM) from 2D and 3D culture platform was generated from similarly cultured MSC without PEMF treatment $(0 \mathrm{mT})$.

In either culturing platform, $\mathrm{CM}$ was collected and subsequently concentrated $10 \times$ by high centrifugation force using a protein concentrator with a molecular weight cut-off of $3 \mathrm{kDa}$ (Thermo Fisher Scientific, USA). In subsequent experiments, the concentrated $\mathrm{CM}$ was diluted 1:10 with low-glucose DMEM media without FBS prior to application to the experimental culture to achieve a final working strength of $1 \times \mathrm{CM}$.

\section{MSC chondrogenesis}

Chondrogenic differentiation of MSCs was induced in 3D pellet cultures as previously described $[45,46]$. Briefly, $2.5 \times 10^{5}$ cells were centrifuged to form pellets and cultured in a chondrogenic differentiation medium containing high-glucose DMEM supplemented with $4 \mathrm{mM}$ proline, $50 \mu \mathrm{g} / \mathrm{mL}$ ascorbic acid, 1\% ITS-Premix (Becton-Dickinson, San Jose, CA), $1 \mathrm{mM}$ sodium pyruvate, and $10^{-7} \mathrm{M}$ dexamethasone (Sigma Aldrich, St Louis, MO), for up to 7 or 21 days in the presence of $10 \mathrm{ng} / \mathrm{mL}$ of transforming growth factor- $\beta 3$ (TGF $\beta 3$; R\&D Systems, Minneapolis, $\mathrm{MN})$. Streptomycin and penicillin were excluded from the 
chondrogenic differentiation media to avoid interference with TRPC channel gating [34, 35]. To study the chondrogenic potential of the MSC secretome, the chondrogenic media, in the absence or presence of TGF $\beta 3$, was supplemented with CM.

\section{Chondrocyte redifferentiation}

Chondrocytes were isolated from pig (animal not directly involved in this study) articular cartilage following enzymatic digestion as previously described [47]. Isolated chondrocytes were expanded in low-glucose DMEM with 10\% FBS without antibiotics and used at passage 1 for all the experiments. Chondrogenic redifferentiation was induced in 3D pellet cultures in the presence of $10 \mathrm{ng} / \mathrm{mL}$ of TGF $\beta 3$. Briefly, $2 \times 10^{5}$ chondrocyte cells were centrifuged to form pellets and kept overnight in expansion media at $37^{\circ} \mathrm{C}$ in $5 \% \mathrm{CO}_{2}$ atmosphere. To study the chondrogenic potential of the MSC secretome for chondrocyte redifferentiation, the expansion media was replaced with chondrogenic media supplemented with the CM and cultured up to 7 or 21 days with medium change every $2-3$ days.

\section{Cell migration}

The migration of MSCs and chondrocytes in response to $\mathrm{CM}$ was assessed using a 24-well Transwell culture ( $8 \mu \mathrm{m}$ pore size, Millipore, Germany). Briefly, MSCs $(3 \times$ $10^{4}$ ) or chondrocytes $\left(5 \times 10^{4}\right)$ were suspended in $300 \mu \mathrm{l}$ of low-serum culture medium (DMEM supplemented with $0.5 \%$ FBS (Life Technologies)) and placed into the upper chamber, and CM was added to the lower chambers of the Transwell culture, containing DMEM with $0.5 \%$ FBS. After $16 \mathrm{~h}$, the upper surface of the Transwell filters was swabbed to remove cells. Cells on the underside of the filter, representing the migrated cells, were then fixed in $4 \%(\mathrm{v} / \mathrm{v})$ paraformaldehyde and stained with hematoxylin and eosin (Sigma Aldrich). The cells in five randomly selected fields at $40 \times$ magnification were counted to indicate migrated cells.

\section{Inflammatory induction of MSCs and chondrocytes}

MSCs or chondrocytes were plated at $1.5 \times 10^{4}$ or $3 \times 10^{4}$ cells/well, respectively, in a 24-well plate in DMEM containing $10 \%$ FBS. IL-1 3 (5 ng/ml; RnD systems) was added to the expansion media at $24 \mathrm{~h}$ after cell seeding to simulate inflammatory conditions. To investigate the inflammation modulatory effect of MSC secretome, CM was added to the culture $24 \mathrm{~h}$ after the induction of inflammation. MSCs or chondrocytes, induced with IL-1 $\beta$, without subsequent $\mathrm{CM}$ treatment served as inflammation (positive) controls, whereas non-inflammation (negative) controls consisted of MSCs or chondrocytes without the addition of IL-1 $\beta$ and CM treatments. Cells and media were harvested 24 and $48 \mathrm{~h}$ post-supplementation with $\mathrm{CM}$ for RNA and nitric oxide synthase (NOS) analysis. NOS activity in the media was analyzed with a NOS assay kit (Abcam, USA). Real-time PCR analysis was performed on the harvested cells to assess the inflammation modulation as a result of the CM.

For post-chondrogenic inflammation induction, MSC pellets were administered IL- $1 \beta(5 \mathrm{ng} / \mathrm{ml})$ for $24 \mathrm{~h}$ before being supplemented with $\mathrm{CM}$. The inflammation modulatory effect of the CM with reference to MSC-derived chondrogenesis was investigated by real-time PCR analysis at day 7 of differentiation.

\section{Cell proliferation and apoptosis}

To assess cell proliferation DNA was analyzed using Quant-iT $^{\mathrm{Tm}}$ PicoGreen $^{\mathrm{Tw}}$ dsDNA Assay Kit (Life Technologies) over a period of 3 days. For determination of antiapoptotic capacity of CM, MSCs or chondrocytes were seeded at $1.5 \times 10^{4}$ or $3 \times 10^{4}$ cells/well in a 24-well plate and treated with Staurosporin $(200 \mathrm{nM}$, Sigma Aldrich) for $2 \mathrm{~h}$ in the presence of CM. The extent of apoptosis was indicated by Caspase 3/7 activity using a Caspase 3/7 assay kit (Promega, Singapore).

\section{Real-time PCR analysis}

Total RNA was extracted using the RNeasy ${ }^{\circ}$ Mini Kit (Qiagen, Germany). Reverse transcription was performed with 100 ng total RNA using iScript ${ }^{\text {mat }}$ cDNA synthesis kit (Bio-Rad, USA). Real-time PCR was conducted using the SYBR $^{\circ}$ green assay on ABI Step One Plus Real-Time PCR System (Applied Biosystems, Life Technologies, USA). Real-time PCR program was set at $95^{\circ} \mathrm{C}$ for 10 min, followed by 40 cycles of amplifications, consisting of a $15 \mathrm{~s}$ denaturation at $95^{\circ} \mathrm{C}$ and a $1 \mathrm{~min}$ extension step at $60^{\circ} \mathrm{C}$. The human and porcine primer sequences used in this study are listed in Additional file 1: Table S1. The level of expression of the target gene, normalized to GAPDH, was then calculated using the $2^{-\Delta \Delta C t}$ formula with reference to the undifferentiated MSC. Results were averaged from triplicate samples of two independent experiments.

\section{ECM and DNA quantification}

Samples harvested were digested with $10 \mathrm{mg} / \mathrm{mL}$ of pepsin in $0.05 \mathrm{M}$ acetic acid at $4{ }^{\circ} \mathrm{C}$, followed by digestion with elastase $(1 \mathrm{mg} / \mathrm{mL})$. A Blyscan sulfated glycosaminoglycan (sGAG) assay kit (Biocolor Ltd., Newtownabbey, Ireland) was used to quantify sGAG deposition according to manufacturer's protocol. Absorbance was measured at $656 \mathrm{~nm}$, and sGAG concentration was extrapolated from a standard curve generated using a sGAG standard. Type II Collagen (Col 2) content was measured using a captured enzyme-linked immunosorbent assay (Chondrex, Redmond, WA). Absorbance at $490 \mathrm{~nm}$ was measured and the concentration of Col 2 was extrapolated from a standard curve generated 
using a Col 2 standard. Values for sGAG and Col 2 content obtained were normalized to the total DNA content of respective samples, measured using Picogreen dsDNA assay (Molecular Probes, OR, USA). Quadruplicates of each group were analyzed from two independent experiments.

\section{Secretome analysis}

A RayBio fluorescent antibody array (Genomax Technologies, SG) was customized for analyzing the secretome of MSC. The CM of 2D cultured MSCs without $(0 \mathrm{mT})$ and with PEMF exposure at $3 \mathrm{mT}$ for $10 \mathrm{~min}$ were concentrated $10 \times$ using a protein concentrator with a molecular weight cut-off of $3 \mathrm{kDa}$ (Thermo Fisher Scientific, USA). The staining of the arrays was performed according to the manufacturer's protocol. Images were acquired using a GenePix 4000B microarray scanner and analyzed with GenePix Pro software (Molecular Devices, USA) for the relative fluorescent intensities of the customized protein targets.

\section{Statistical analysis}

All experiments were performed in biological replicates $(n=3)$ and results reported as mean \pm standard deviation (SD). Statistical analysis was carried out by Student's $t$ test for comparison between two groups using the Microsoft Excel software. The level of significance was set at $p<0.05$. All quantitative data reported were averaged from at least two independent experiments.

\section{Results}

\section{Chondrogenic potential of PEMF-conditioned media generated from MSCs in 3D culture}

We have previously shown that MSCs in pellet culture (3D) exhibited an enhancement in chondrogenic induction when exposed to PEMFs at a discrete efficacy window of $2 \mathrm{mT}$ applied once for $10 \mathrm{~min}$ [34]. Here we show that the MSC secretome contributes to the chondrogenic potential of PEMF exposure. The characteristic PEMFinduced upregulations of $\mathrm{Col}$ 2, Aggrecan, and Sox9 were attenuated by the removal of PCM and its replacement with age-matched media harvested from naïve (CM-deprived; CM-dep) unexposed sister cultures $24 \mathrm{~h}$ following PEMF exposure (Fig. 2), serving as a manner to selectively deprive cells of PEMF-mediated paracrine stimulation, while leaving other collateral PEMF-dependent responses intact. On the other hand, PEMF-induced chondrogenic induction could be transferred to naive MSCs with the transfer of CM harvested from PEMF-treated MSCs (PCM) $24 \mathrm{~h}$ after exposure. Moreover, PCM acted synergistically with the effects of direct PEMF exposure. The results indicate that the chondrogenic attributes of PEMF exposure is partially mediated through the paracrine activity of the MSC secretome.
MSC pellet cultures were exposed to PEMFs of varying amplitudes and durations and the generated PCMs were transferred to naïve (unexposed) MSC pellet cultures undergoing chondrogenic induction. PCM generated from MSC pellets exposed at $2 \mathrm{mT}$ for $10 \mathrm{~min}$ produced the greatest induction of chondrogenic markers ( $\mathrm{Col} 2$, aggrecan, Sox9) in naïve MSC pellets (Fig. 3a), matching our previously described EMF efficacy window [34]. Conversely, the expression ratios of type X collagen ( $\mathrm{Col} \mathrm{10),}$ alkaline phosphatase (ALP) and matrix metallopeptidase 13 (MMP13) to Col 2, indices of chondrogenic hypertrophy, were most strongly suppressed by PCM generated at the peak of the efficacy window ( $2 \mathrm{mT}$ for $10 \mathrm{~min}$ ).

TGF 33 is commonly used to facilitate chondrogenic induction $[45,46]$. Notably, PCM generated at $2 \mathrm{mT}$ for 10 min was capable of promoting chondrogenesis in the absence of TGF 33 by four to fivefold. In the presence of 10 $\mathrm{ng} / \mathrm{ml}$ TGF $\beta 3$, chondrogenic induction was further enhanced by PCM exhibiting increases of $>10$-fold for both Col 2 and aggrecan. Sox 9 expression levels were more modestly enhanced with PCM generated at $2 \mathrm{mT}$ for 10 min in the absence $(\sim 3$-fold) or presence $(\sim 4$-fold) of TGF $\beta$. By contrast, PCM harvested from 3D MSC cultures that were exposed to PEMFs of greater amplitudes or longer durations did not exhibit any significant effects relative to pellets administered control CM (CCM, $0 \mathrm{mT}$ ). The inhibition of chondrogenic hypertrophy by PCM harvested at peak PEMF amplitude $(2 \mathrm{mT})$ was also preserved in the presence of TGF 33 (Fig. 3a).

The chondrogenic enhancement afforded by PCM administration at the transcriptional level was further corroborated at the protein level, whereby the PCM obtained from MSC 3D pellet cultures following exposure to $2 \mathrm{mT}$ PEMFs produced significant increases in both $\mathrm{Col} 2$ and sGAG proteins with an associated increase in cellular DNA content relative to CCM (Fig. 3b).

\section{Chondrogenic potential of PCM generated from MSCs in 2D culture}

It is well established that the availability and nature of cellular-substrate interactions influence the paracrine activity of MSCs $[18,23,48]$. We next assessed the chondrogenic potential of PCM harvested from MSCs grown in 2D cultures. MSCs cultured on the surface of tissue culture dishes were exposed to PEMF amplitudes ranging from 0 to $4 \mathrm{mT}$ for single $10 \mathrm{~min}$ exposures and the generated $\mathrm{CMs}$ were then transferred to naive (unexposed) MSC pellet cultures undergoing chondrogenic induction in the presence of TGF $\beta$. PCM harvested from MSCs exposed to $3 \mathrm{mT}$ for $10 \mathrm{~min}$ produced the greatest upregulations of Col $2(\sim 17$-fold $)$ and aggrecan ( 4-fold) expression in naive MSC pellets, whereas PCMs generated from MSCs exposed at PEMF amplitudes other than $3 \mathrm{mT}$ or for longer exposures $(30 \mathrm{~min})$ produced no additional chondrogenic 

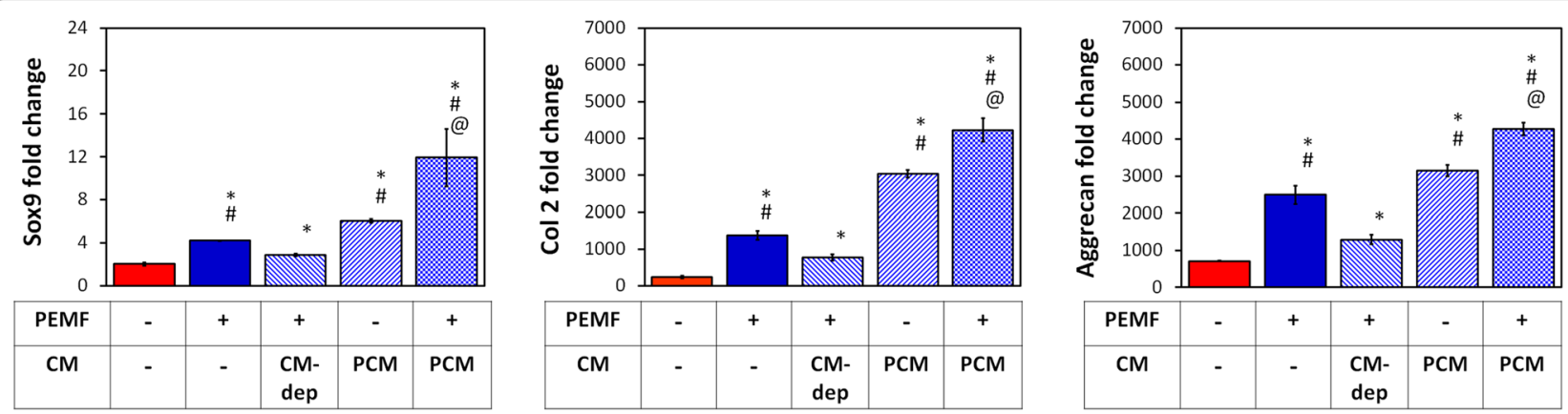

Fig. 2 Brief PEMF exposure stimulates MSC-derived paracrine activity to promote chondrogenesis. MSCS in 3D pellet cultures undergoing chondrogenesis were subjected to PEMFs at either 0 or $2 \mathrm{mT}$ for $10 \mathrm{~min}$. The conditioned media generated after $24 \mathrm{~h}$ of PEMF exposure (PCM) was either replaced with age-matched media from unexposed sister cultures (CM-dep; CM-deprived), or transferred to age-matched exposed or unexposed sister cultures. Real-time PCR analysis of cartilaginous markers were performed after 7 days of differentiation and normalized to GAPDH. Results were presented as fold-changes relative to the level in undifferentiated MSCs. Data shown are means \pm SD, $n=6$ from 2 independent experiments. ${ }^{*}$ denotes significance differences compared to non-PEMFed controls (red bars); \# denotes significance differences compared to CM-deprived (CM-dep) condition; @ denotes significance differences compared to treatment with PCM alone

enhancement relative to MSC pellets treated with control CM (CCM, $0 \mathrm{mT}$ ) (Fig. 4a). As with 3D MSC-generated $\mathrm{PCM}$, downregulations in the expression of the hypertrophic markers Col 10, ALP, and MMP13 were most apparent with peak PCM (3 mT) compared to CCM (Fig. 4a). The chondrogenic enhancement apparent at the transcriptional level with PCM generated from 2D MSCs exposed to $3 \mathrm{mT}$ for $10 \mathrm{~min}$ was also corroborated at the protein level, with a twofold increase in Col 2 and sGAG deposition compared to non-PEMFed CCM (Fig. 4b). Accordingly, no increase in ECM deposition was detected with PCM generated at off peak conditions.

\section{Effects of PCM on chondrocytes}

We next investigated the chondrogenic effects of the MSC-PCM on chondrocyte redifferentiation. Chondrocytes as pellet cultures in the presence of TGF $\beta$ were treated with PCM generated from MSCs cultured in either 2D or 3D platforms exposed at their respective peak amplitudes of $3 \mathrm{mT}$ and $2 \mathrm{mT}$. Both PCMs better sustained the chondrogenic phenotype in redifferentiating chondrocytes, relative to their respective CCM. Increased expression of $\mathrm{Col} 2$ and aggrecan (1.5 to 2-fold) was detected, whereas decreases in the expression of $\mathrm{Col}$ 1 and Col 10 were observed, relative to the respective CCM (Fig. 5a). The relative efficacy of either PCM at its associated peak amplitude and platform on primary chondrocytes was further corroborated at the protein level for both Col 2 (> 3-fold increase) and sGAG ( 2fold increase) production (Fig. 5b). The results suggest that PCMs harvested from either 2D or 3D MSC cultures at their respective peak amplitudes and exposure durations were capable of offsetting chondrocyte dedifferentiation and promote the expression of hyaline cartilage markers.

\section{Effect of PCM on chondrocyte and MSC migration and proliferation}

We assessed if 2D MSC-generated PCM could affect the migration and proliferation of chondrocytes and MSCs, the two cell types localized in the articular joint environment that participate in cartilage regeneration $[8,49]$. CCM and PCM enhanced cell migration of both chondrocytes and MSCs relative to respective negative controls (Fig. 6a). PCM, however, produced a further twofold and fourfold increase in migratory capacity for chondrocytes and MSCs, respectively, relative to the CCM, approaching the migration level observed for the positive controls (10\% FBS). Chondrocyte proliferation was enhanced to similar magnitudes with either CCM or PCM, whereas MSC proliferation was largely unaffected by either $\mathrm{CM}$ under the presented culturing conditions (Fig. 6b). PCM thus appears to hold potential for enhancing MSC-derived paracrine-dependent chemotaxis of MSCs and chondrocytes.

\section{Anti-inflammatory effects of PCM on chondrocytes and MSCs}

The MSC secretome has been ascribed antiinflammatory properties [14, 29]. We investigated the potential immunomodulatory attributes of 2D MSCgenerated PCM over inflammation-induced chondrocytes. Primary chondrocytes treated with IL- $1 \beta$ exhibited attenuated expression of the ECM markers, Col 2 and aggrecan, coincident with an increased expression of the pro-inflammatory markers, IL-6, MMP13, and COX-2, as well as enhanced NOS activity (Fig. 7). Although NOS activity was suppressed by either PCM or CCM relative to the inflammatory control (IL-1 $\beta$ treatment), PCM exerted an additional $\sim 2-3$-fold greater suppression of NOS compared to CCM that was sustained throughout the 48-h examination period. Suppressions 


\section{A $\quad(-)$ TGF- $\beta$}
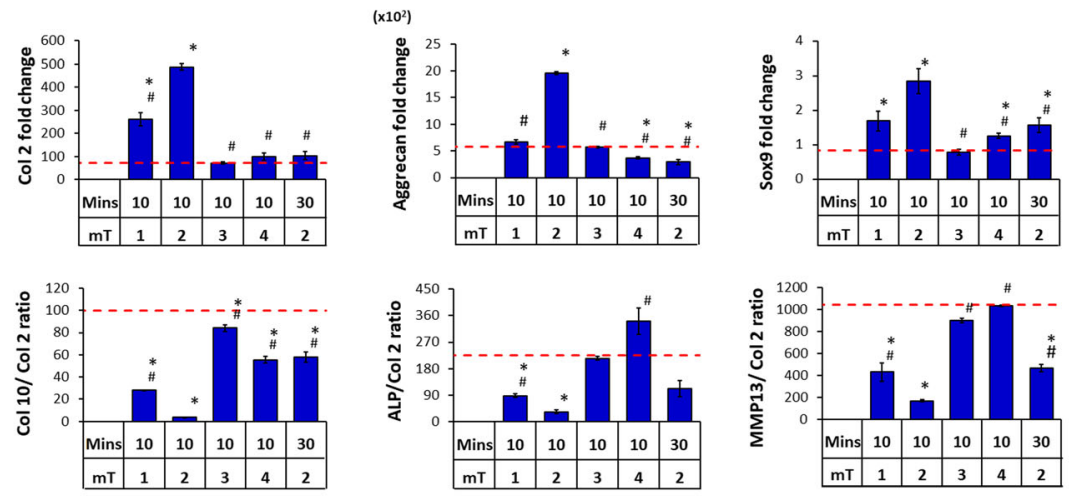

$(+)$ TGF- $\beta$
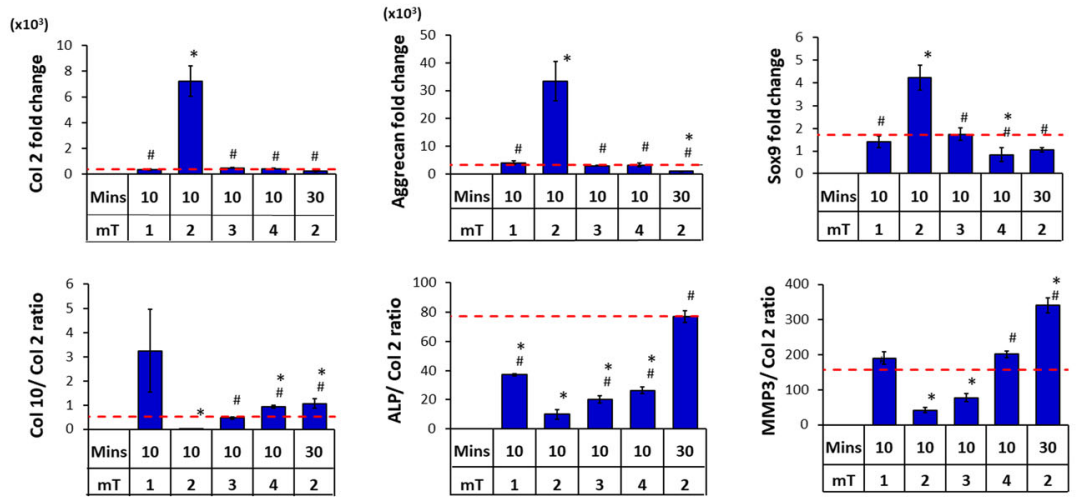

B
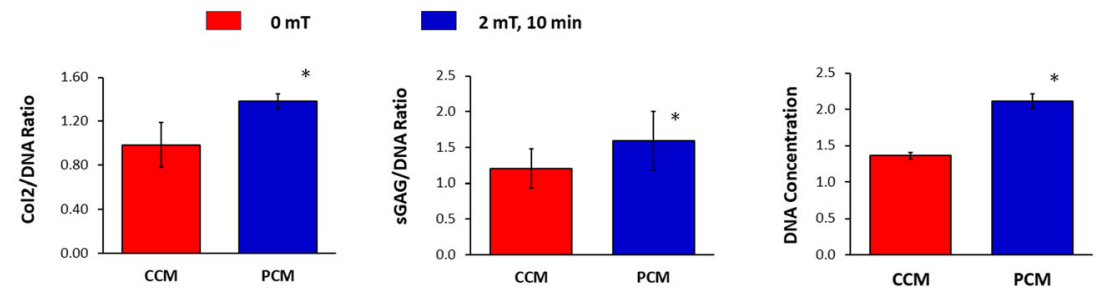

Fig. 3 Effect of PEMF-induced conditioned media (CM) from 3D cultured MSCs on chondrogenic differentiation of MSCs. MSCs in 3D culture were subjected to PEMF exposure at different intensities and durations. The generated CM was collected $24 \mathrm{~h}$ post-PEMF exposure and tested for chondrogenic effect over naive (unexposed) MSC pellet cultures undergoing chondrogenic differentiation in the absence or presence of TGF $\beta$. a Real-time PCR analysis of cartilaginous and hypertrophic marker expression after 7 days of differentiation normalized to GAPDH and presented as fold-changes relative to levels in undifferentiated MSCs. The expression of hypertrophic markers was presented as the ratio to Col 2 expression. $\mathbf{b}$ Quantification of cartilaginous extracellular matrix macromolecules generated by the differentiated MSCs (+TGF $\beta$ ) after 21 days of differentiation. Data shown represent means $\pm S D, n=6$ from 2 independent experiments. * denotes significance differences compared to non-PEMFed (CCM, $0 \mathrm{mT}$ ) controls (red dash lines or red bars). \# denotes significance differences compared to PEMFed CM (PCM) generated at $2 \mathrm{mT}, 10 \mathrm{~min}$

in the levels of IL-6 (> 1.5- and 2.5-fold), MMP-13 (10and 13-fold), and COX-2 (> 2.5- and 2-fold) were also observed commencing at $24 \mathrm{~h}$ after administration of either CCM or PCM, respectively. Notably, chondrocytes exhibited elevated basal levels of IL- 6 and COX- 2 in the noninflammation controls (no IL- $1 \beta$ treatment), indicating significant levels of resting inflammation in chondrocytes. Reinstated expression of chondrogenic markers ( $\mathrm{Col} 2$ and aggrecan) was observed following administration of the
PCM at 24 and $48 \mathrm{~h}$ relative to the inflammatory control and CCM (Fig. 7) that, although were mitigated relative to the non-inflammatory controls, were showing a trend towards recovery at $48 \mathrm{~h}$.

Similar to chondrocytes, IL-1 $\beta$ treatment elicited a state of inflammation in MSCs that was evidenced by upregulations in IL-6, MMP-13, COX-2, and nitric oxide synthase (NOS) (Fig. 8a). By contrast, MSCs were overall more resistant to developing IL-1 $\beta$-induced inflammation as 


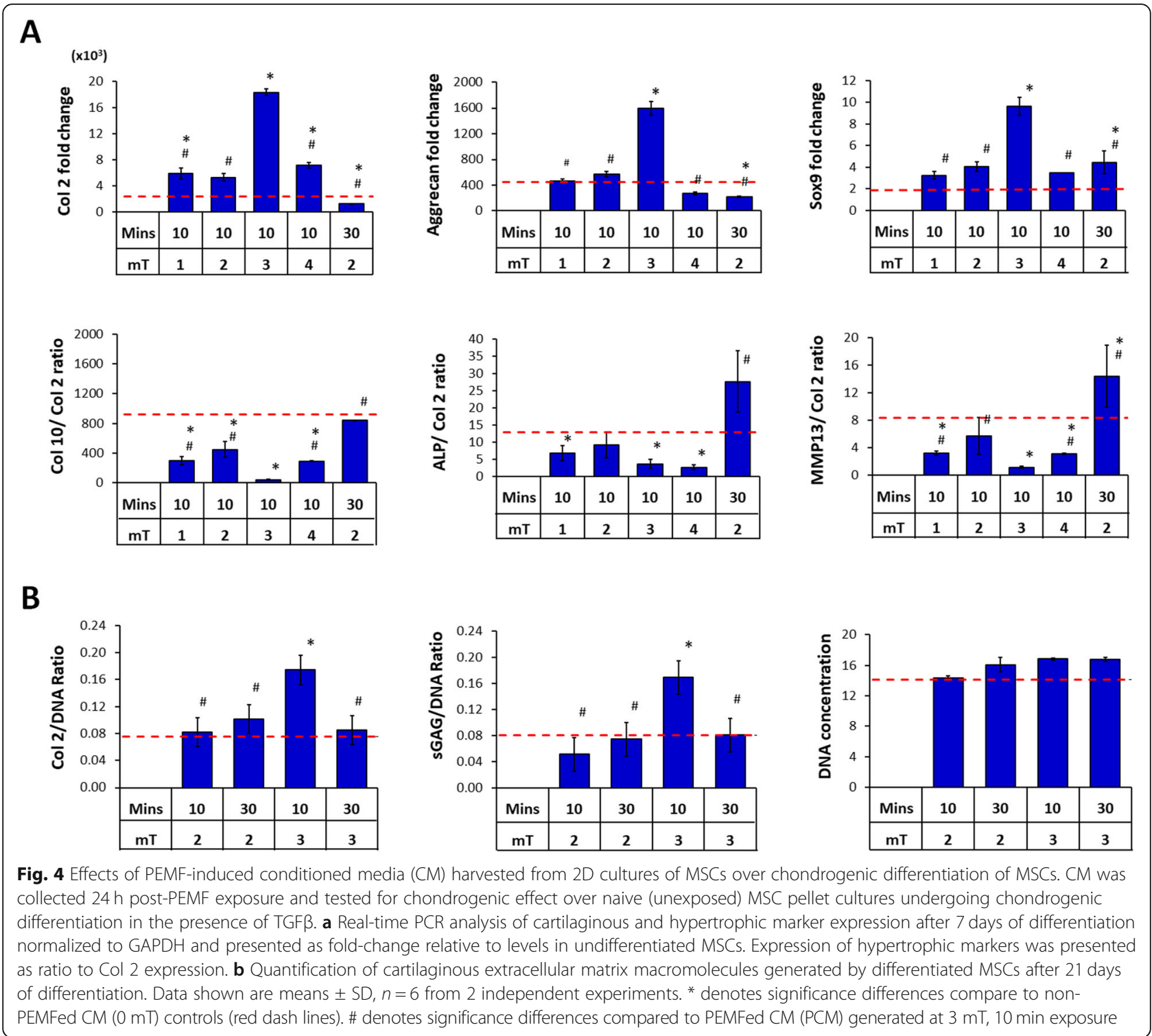

appreciable levels of inflammatory markers were generally only achieved after $48 \mathrm{~h}$. Reductions in MMP-13 and COX-2 were observed with both CCM and PCM administration at 24 and $48 \mathrm{~h}$ post-administration with overall greater suppression observed with PCM. The levels of COX-2 showed significant reductions at both 24 and $48 \mathrm{~h}$ upon administration of PCM, particularly with reference to non-inflammatory controls (no IL-1 $\beta$ treatment). IL-6 levels were suppressed most strongly $48 \mathrm{~h}$ after administration of PCM relative to the inflammatory control (IL$1 \beta$ treatment). PCM administration also produced significant suppressions of IL- $1 \beta$-induced NOS activity at both 24 and $48 \mathrm{~h}$, whereas CCM showed no effect at either time point. Notably, the level of NOS activity observed in response to PCM administration at $48 \mathrm{~h}$ was identical to that in the non-inflammatory control, indicating complete normalization of basal NOS activity.

The effects of PCM over MSC chondrogenesis were also examined under conditions of induced inflammation. IL$1 \beta$ treatment suppressed the expression of the chondrogenic markers, Col 2, aggrecan, and Sox9 (Fig. 8b). Partial rescue of Col 2 (>9-fold and 20-fold), aggrecan (>3-fold and 15-fold) and Sox9 (>2.5-fold and 5.5-fold) was achieved in samples supplemented with either CCM or $\mathrm{PCM}$, respectively, compared to the inflammatory controls (IL-1 $\beta$ treatment). As noted, PCM consistently produced greater levels of protection than CCM. PEMF treatment hence was capable of enhancing MSC-derived paracrine factors capable of attenuating cellular inflammation, partially reinstating MSC chondrogenesis. 


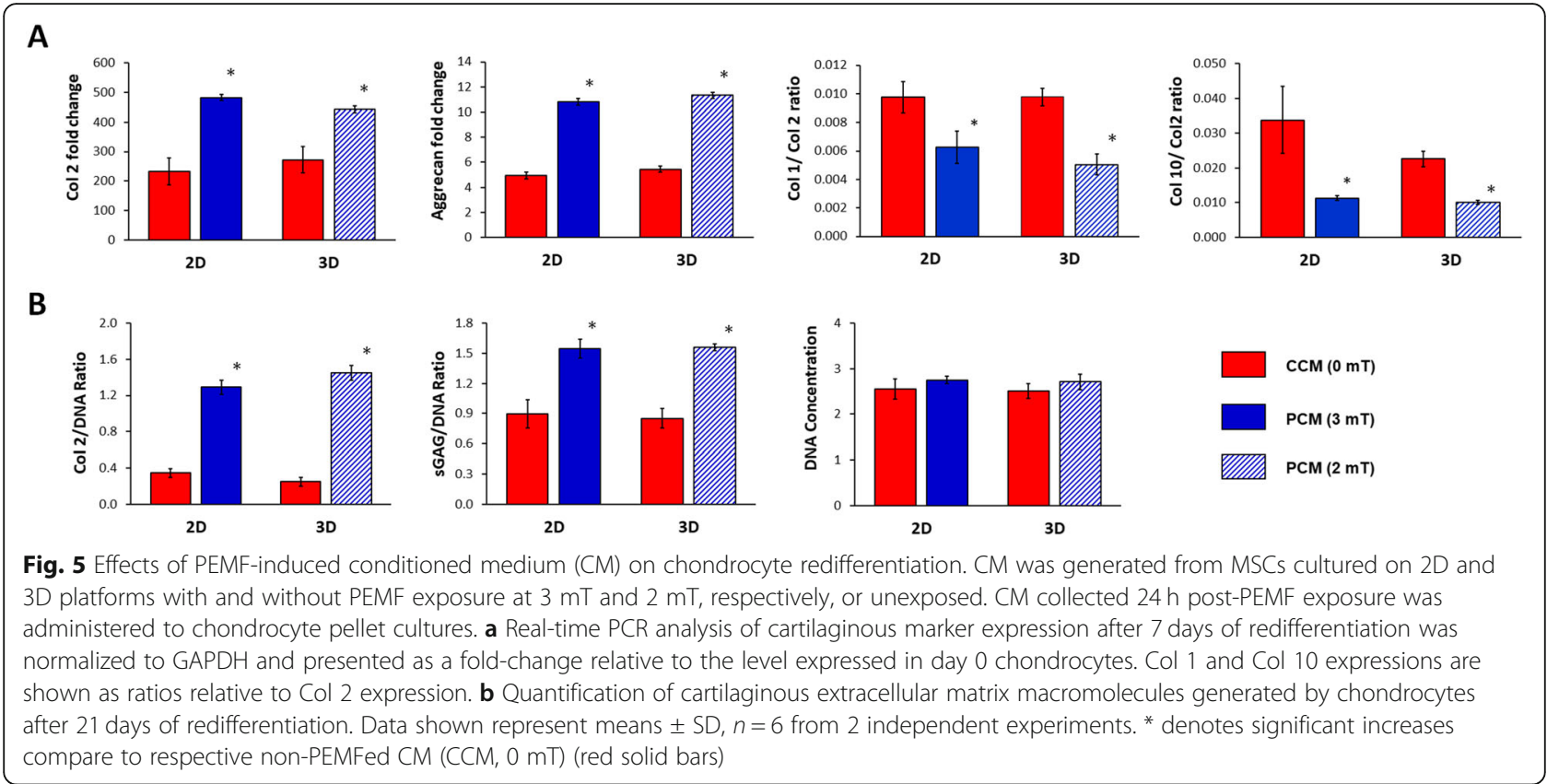

A
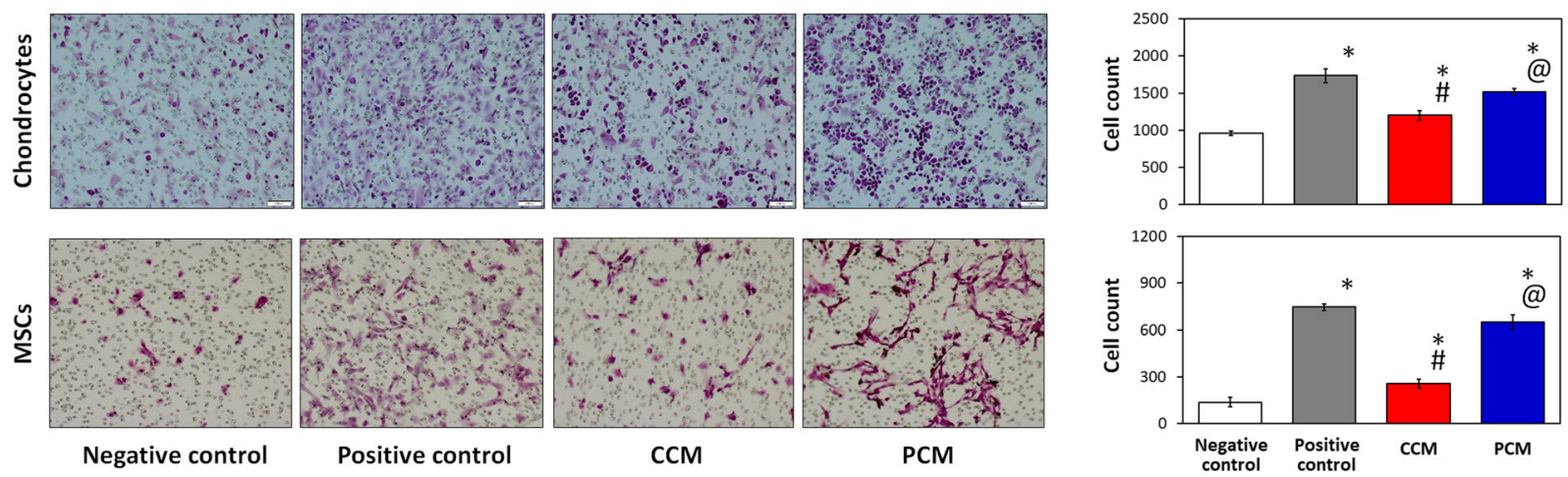

B
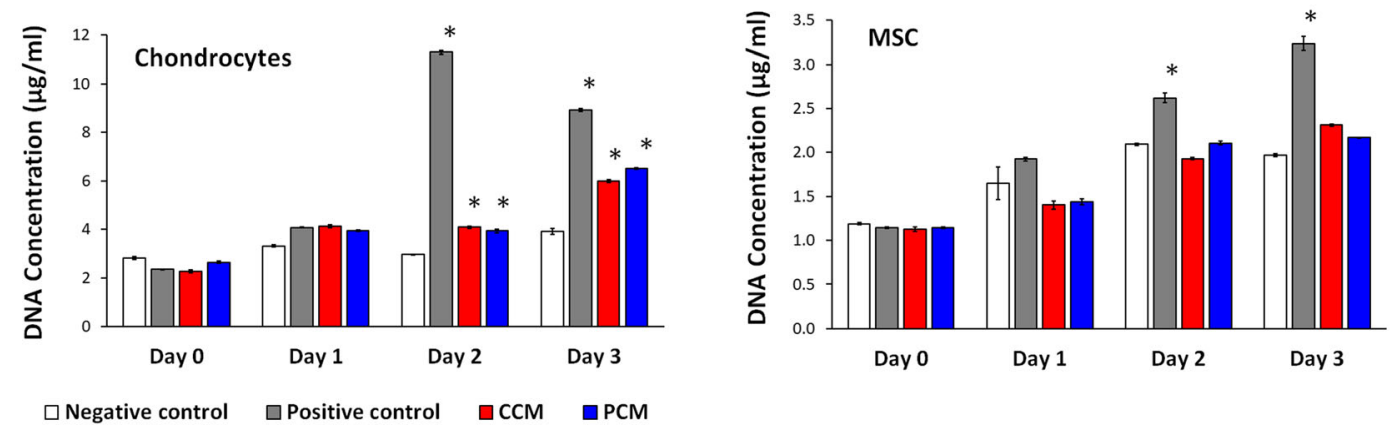

Fig. 6 Effect of PEMF-induced conditioned medium (CM) on chondrocyte and MSC migration and proliferation. CM was derived from 2D cultured MSCs in response to $0 \mathrm{mT}$ (CCM) or $3 \mathrm{mT}$ (PCM) PEMF exposure. a Migration of chondrocytes or MSCs was analyzed using a transwell culture. Migrated cells were assessed by measuring the number of cells on the underside of the transwell filter after H\&E staining. $\mathbf{b}$ Cell proliferation was determined by Picogreen DNA Assay. Data shown represent means \pm SD, $n=6$ from 2 independent experiments. * denotes significant difference compared to the negative control (Expansion media + 0.5\% FBS); \# denotes significant differences compared to positive controls (Expansion media + 10\% FBS); @ denotes significant differences compared to the non-PEMFed CCM 


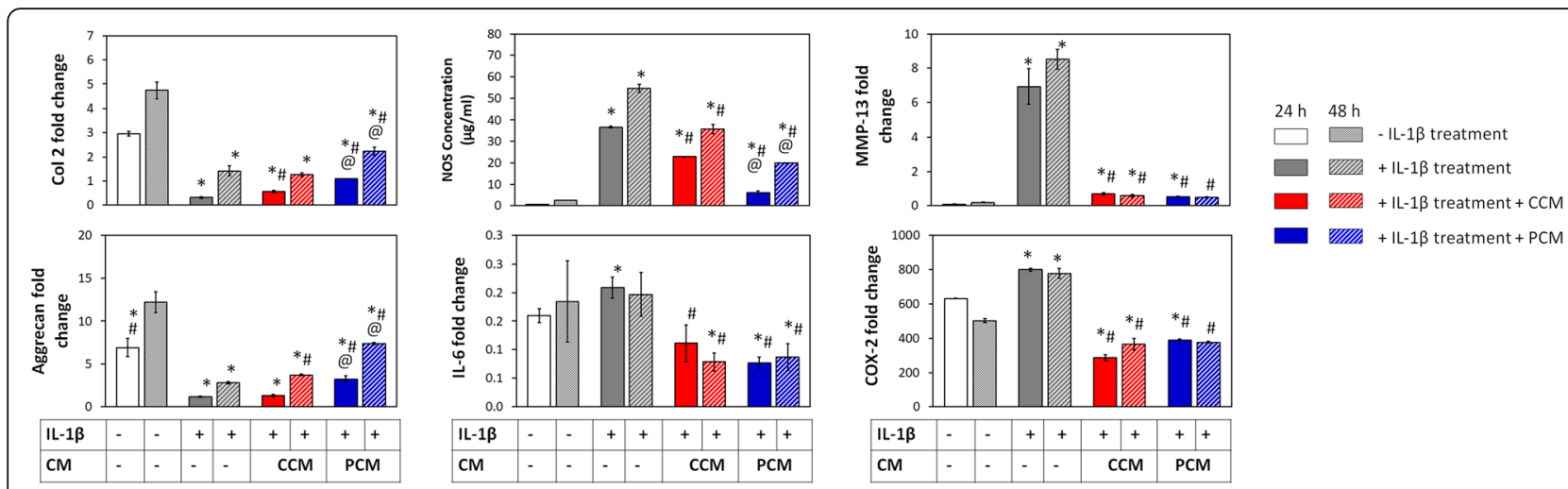

Fig. 7 Effect of PEMF-induced conditioned medium (CM) on inflamed chondrocytes. Inflammation was induced in chondrocytes with $5 \mathrm{ng} / \mathrm{ml} \mathrm{IL}-$ $1 \beta$ for $24 \mathrm{~h}$. CCM or PCM was administered to chondrocytes $24 \mathrm{~h}$ post inflammation induction. Real-time PCR analysis of cartilaginous and inflammatory markers and NOS activity were performed at $24 \mathrm{~h}$ (plain bars) and $48 \mathrm{~h}$ (hatched bars) post-supplementation with CM and normalized to GAPDH, presented as a fold-change relative to the level in non-treated (day 0 ) chondrocytes. Data shown represent means \pm SD, $n=6$ from 2 independent experiments. * denotes significant differences compared to the non-inflamed controls (no IL-1 $\beta$ treatment); \# denotes significant differences compared to the inflammation controls (IL-1 $\beta$ alone treatment) and @ denotes significant differences compared to respective CCM. Col 2 = type II collagen; COX-2 = cycloxigenase-2; IL-1 $\beta=$ interleukin-1 $\beta$; IL-6 = interleukin-6; MMP-13= metalloproteinase 13; NOS = nitric oxide synthase

\section{Effect of PCM on chondrocyte and MSC apoptosis}

We have previously shown that brief $(10 \mathrm{~min})$ exposure to PEMFs was capable of attenuating basal apoptosis in myoblasts during early myogenesis [35]. We evaluated the potential of 2D MSC-generated CM to modulate apoptosis in chondrocytes and MSCs subjected to Staurosporin $(200 \mathrm{nM})$. Basal caspase activity was elevated in control chondrocytes reflecting the elevated resting inflammation demonstrated previously (Fig. 7) and could be further augmented with exposure to Staurosporin. PCM significantly suppressed caspase activity in chondrocytes after Staurosporin treatment, effectively normalizing basal apoptosis levels (Fig. 9a). By contrast, no antiapoptotic effect was observed in chondrocytes administered CCM relative to the Staurosporin-treated control. MSCs in which apoptosis was induced with Staurosporin and supplemented with either CCM or PCM exhibited a comparable and significant decrease in caspase activity relative to the Staurosporin-treated control (Fig. 9b). PEMF treatment hence potentiates the paracrine-mediated attenuation of apoptosis of MSC $\mathrm{CM}$ in chondrocytes that could ultimately translate to improved regenerative responses.

Expression and secretion of paracrine factors modulating differentiation, proliferation, migration, and inflammation from PEMF-treated MSCs

The ability of PEMFs to influence the production and release of factors associated to chondrogenic differentiation, proliferation, migration, and inflammatory modulation was examined from 2D cultures of MSCs. Factors examined included bone morphogenic protein (BMP), transforming growth factor (TGF), thrombospondin (TSP), insulin-like growth factor (IGF), COX-2, IL-10, and interleukin 1 receptor antagonist (IL-1ra). The expression of BMP2, BMP4, TSP-2, IL-1ra, and IL-10 were upregulated by $\sim 2$-fold, or greater, $24 \mathrm{~h}$ after PEMF treatment, relative to non-exposed MSCs. TGF $\beta 1$, TGF $\beta 3$, IGF-2, and COX2 exhibited less significant increases, whereas TSP-1 showed no change in expression (Fig. 10a).

Antibody arrays were used to characterize the secretion profile of the MSC CM. PEMF exposure at $3 \mathrm{mT}$ promoted the secretion of BMP-2, BMP-4, TSP-2, and IL-1ra (Fig. 10b), consistent with observed increases in gene expression (Fig. 10a). By contrast, the protein secretion of TGF $\beta 1$ and IL10 was dampened by PEMF treatment, while TGF $\beta 3$ and TSP-1 secretion was not affected, reflecting transcriptional to translation temporal disparity.

\section{Discussion}

MSCs secrete a plethora of bioactive factors, which through paracrine means, synchronize the regenerative responses of neighboring cellular communities. For clinical exploitation, strategies to selectively modulate the secretory function of MSCs have been advanced and include subjecting MSCs to a variety of micro-environmental biochemical and mechanical cues, as well as biophysical perturbations $[24,25,50]$. We previously showed that MSC chondrogenic differentiation in 3D pellet cultures could be enhanced by brief exposure to low amplitude and frequency PEMFs administered for $10 \mathrm{~min}$ at an optimal amplitude of $\sim 2 \mathrm{mT}$ [34]. The involvement of transient receptor potential cation (TRP) channels and downstream $\mathrm{Ca}^{2+}$ signaling were implicated in mediating the effects of PEMF exposure. PEMFs were subsequently shown to preferentially activate a TRPC1-mitochondrial axis important for in vitro 


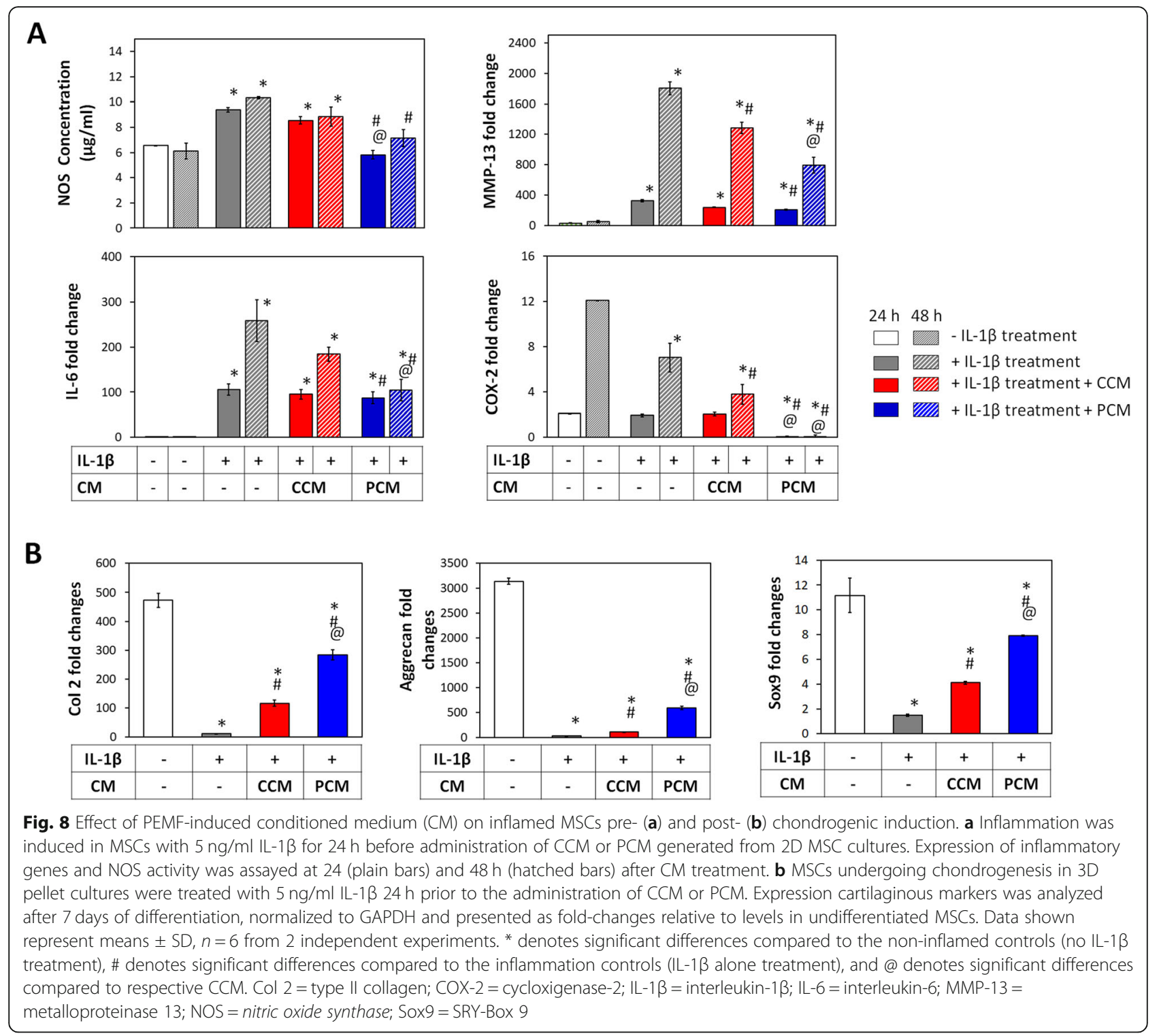

myogenesis and underlying mitochondrial adaptation to oxidative stress via a process of magnetic mitohormesis [35]. Accordingly, activation of mitochondrial respiration is known to activate the muscular secretome [33].

In this study, we examined the effects of brief PEMF exposure on the MSC secretome and its ability to promote MSC-induced chondrogenesis and chondrocyte cartilage tissue formation. The results demonstrated that the capacity of PEMFs to induce MSC chondrogenesis is contributed to by PEMF modulation of MSC secretome. As such, exchange of the bathing medium $24 \mathrm{~h}$ post-PEMF exposure compromised chondrogenesis, clearly indicating the necessity of released paracrine factors (Fig. 2). Harvested CM from PEMF-treated MSCs (PCM) was moreover capable of conferring chondrogenic enhancement onto naïve MSCs and, furthermore, demonstrated synergy when combined with direct PEMF exposure of MSCs. Notably, the PEMF parameters best suited for the production of chondrogenic MSC-PCM from 3D pellets was $2 \mathrm{mT}$ for $10 \mathrm{~min}$ (Fig. 3), identical to the exposure parameters previously determined most efficacious at promoting 3D MSC chondrogenesis in response to direct exposure [34]. PCM generated under this exposure paradigm rendered significant chondrogenic enhancement compared to CCM obtained from non-exposed MSCs. Furthermore, the observed PCM-mediated increase in the expression of cartilaginous matrix was accompanied by a downregulation of hypertrophic markers, Col 10, ALP, and MMP13. The prochondrogenic effect of PCM was also evident in the context of chondrocyte redifferentiation, promoting cartilage formation with superior hyaline phenotype (Fig. 5). Taken together, our data demonstrates that MSCs subjected to 

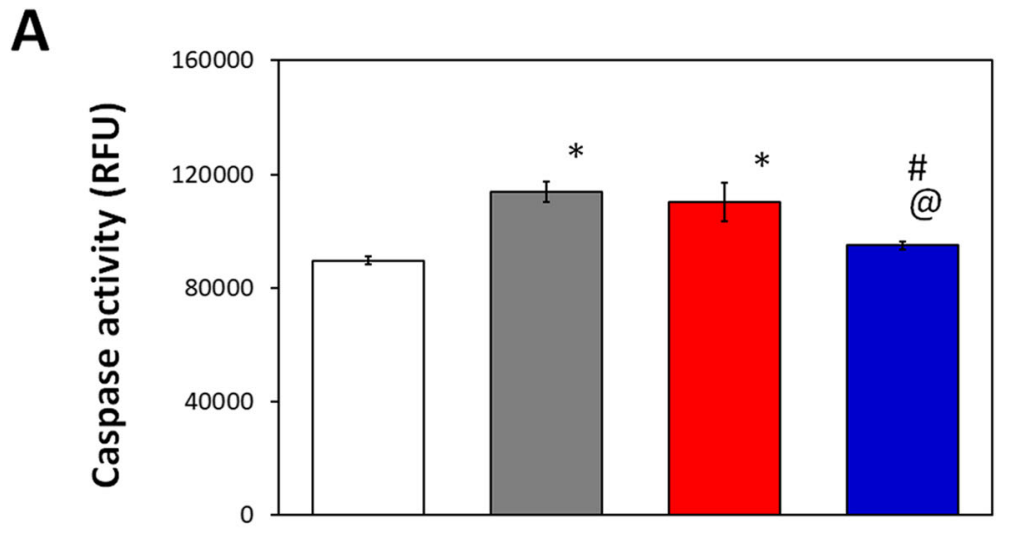

B

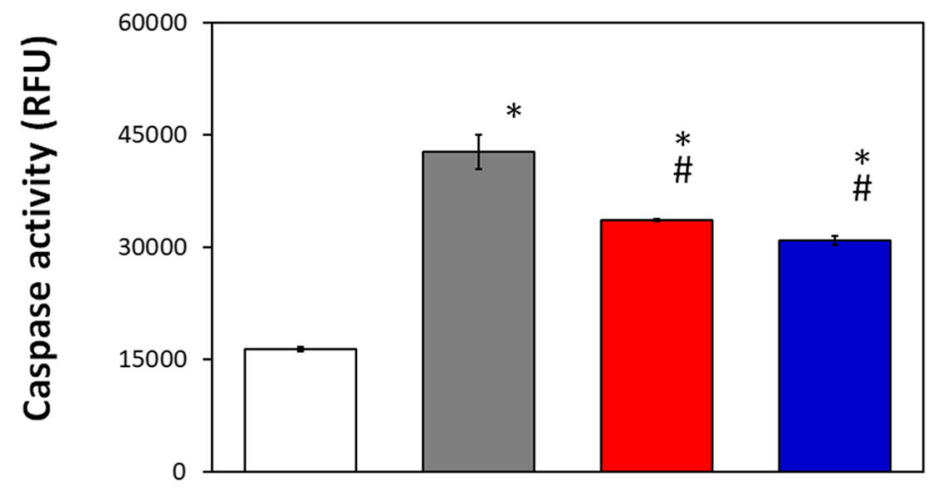

\begin{tabular}{|c|c|c|c|c|}
\hline $\mathrm{SPN}$ & - & + & + & + \\
\hline $\mathrm{CM}$ & - & - & $\mathrm{CCM}$ & $\mathrm{PCM}$ \\
\hline
\end{tabular}

Fig. 9 Effect of PEMF-induced conditioned medium (CM) on the apoptotic status of MSCs and chondrocytes. Chondrocytes (a) or MSCs (b) were treated with Staurosporin (SPN; $200 \mathrm{nM}$ ) in conjunction with supplementation with either CCM or PCM for $2 \mathrm{~h}$. Apoptotic activity was determined by Caspase 3/7 activity and was presented as relative fluorescence units (RFU). Data shown represent means $\pm S D, n=6$ from 2 independent experiments. * denote significant differences compared to no Staurosporin treatment; \# denotes significant differences compared to Staurosporin treatment alone and @ denotes significant differences relative to CCM

chondrogenic induction under conditions of 3D culture respond to PEMF stimulation with secretome modulation that could provide both autocrine and paracrine anabolic effects with ultimate relevance to the in vivo articular scenario.

Components of the MSC secretome, in the form of $\mathrm{CM}$ or as isolated extracellular vesicles (EVs), have demonstrated therapeutic potential in cases of osteochondral lesions $[13,14]$ or in osteoarthritis animal models $[15$, 16]. The therapeutic effect of the MSC secretome has been largely attributed to the multifaceted stimulation of chondrocyte proliferation, migration, cartilaginous ECM generation, and the attenuation of the inflammatory and apoptotic microenvironment associated with injury or joint degeneration $[14-16,51,52]$. The demonstration that the delivery of MSC secretory products was efficacious in cartilage regeneration revealed the possibility of using MSC-derived secretory products as a cell-free therapeutic for joint injury and osteoarthritis. Notably, CM harvested from MSCs in conventional 2D tissue culture had similar chondrogenic potency as 3D PCM in terms of cartilage ECM formation and the capacity to reduce hypertrophic and fibrocartilage development for both MSCs and chondrocytes, although responsive to a distinct, yet higher ( $3 \mathrm{mT}$ for $10 \mathrm{~min}$ ), electromagnetic efficacy window (Figs. 4 and 5). Chondrocyte migration had been previously reported to be stimulated by MSCderived EVs $[14,51]$. Here we show that migration was also enhanced for both chondrocytes and MSCs by PCM (Fig. 6), suggesting the potential for PCM to chemotactically attract chondrocytes or MSCs to the vicinity of an articular injury to promote regeneration.

Following cartilage injury or during osteoarthritis, the expression of inflammatory cytokines and catabolic factors 


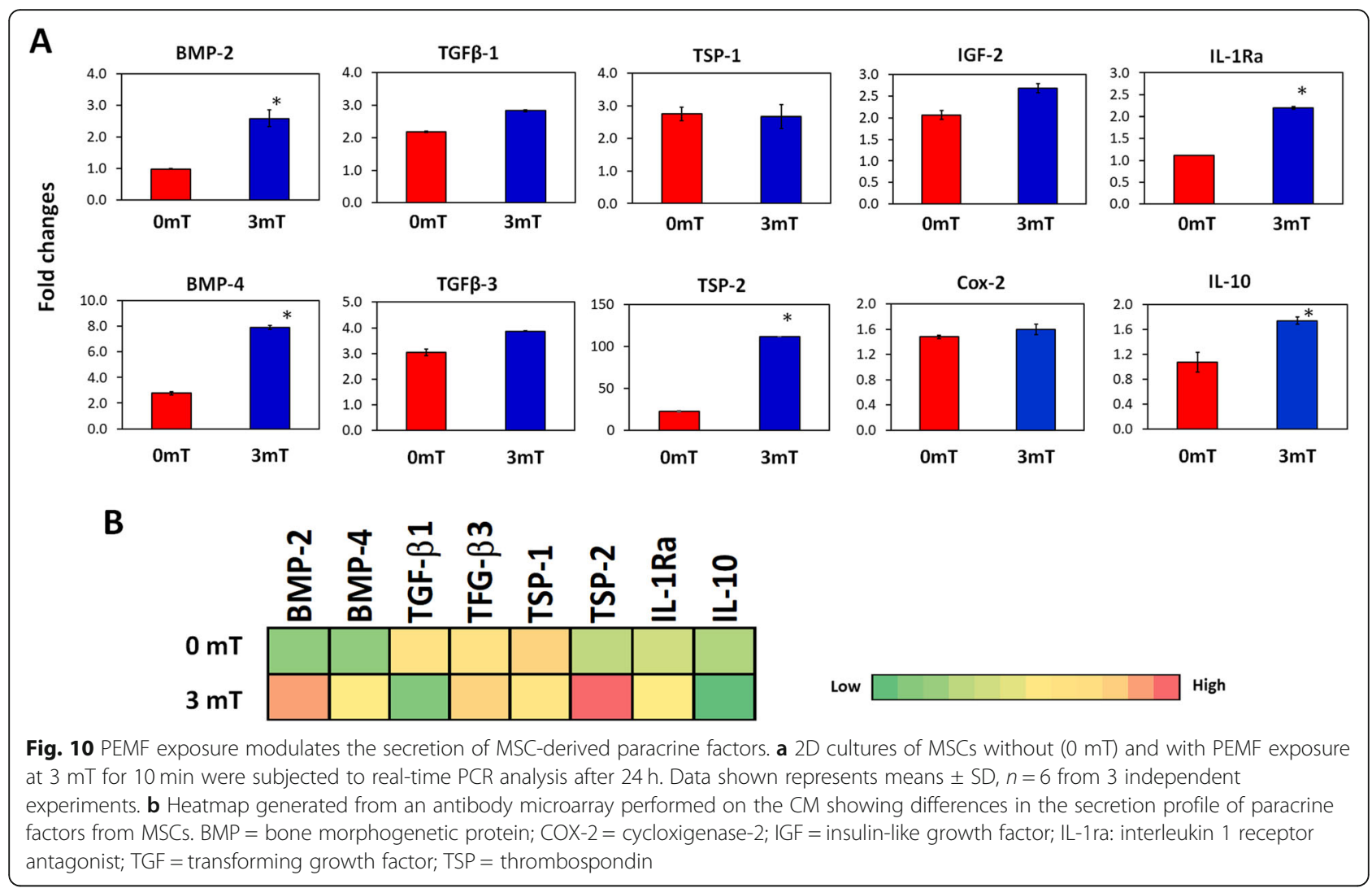

(e.g., matrix metalloproteinases) are upregulated, perpetuating inflammation, cartilage matrix degradation, and chondrocyte apoptosis [53]. MSC-derived EVs have been previously demonstrated to inhibit the adverse effects of inflammatory cytokines on cartilage homeostasis [14-16, 51, 52]. Here we show that the MSC-derived CMs are generally protective against cellular inflammation reflected by suppressions of chondrocyte NOS activity, inflammatory markers (IL-6 and COX-2), and catabolic proteinases (MMP13) (Fig. 7). Significantly, the protective impact of PCM generated from PEMF-exposed MSCs was even greater as revealed by stronger suppressions of NOS activity, preservation of chondrocyte anabolic activities, indicated by upregulating severely suppressed cartilage ECM genes expression (Fig. 7), and rescuing the Staurosporininduced chondrocytes apoptosis (Fig. 9), an effect not observed with non-PEMFed control CM (CCM).

The protection conferred by PCM may hence potentially be extended to an inflamed scenario within the articular environment. Resident MSCs in the joint cavity, arising from either the synovium or bone marrow, in the case of microdrilling/microfracture, have been shown to participate in cartilage regeneration, either by engraftment or as endogenous providers of secreted trophic factors [54, 55]. The regenerative efficacy of resident MSCs, however, would be influenced by the inflammatory environment within the joint. Under conditions of induced inflammation, MSCs experienced increased NOS activity, elevated expressions of IL-6, Cox-2, and MMP13, and attenuated chondrogenic differentiation, degenerative conditions that were effectively suppressed with PCM (Fig. 8) and could potentially be translated to the in vivo scenario. Our results indicate that the brief exposure of MSCs to low-amplitude PEMFs heightens the anti-inflammatory potential of their secretome, alluding to an enhanced therapeutic application to attenuate cartilage damage and restore MSC regenerative capacity in an inflamed articular environment.

MSCs secrete trophic factors including IGF-1, PDGF, FGF, VEGF, and members of the TGF $\beta$ superfamily in response to environmental cues or stimuli $[18,19]$. Accordingly, PEMF-based enhancement of MSC differentiation has been previously linked with the expression and secretion of the TGF and BMP families [36-38]. Moreover, the capacity of PCM to significantly augment cartilaginous ECM production, despite the conventional presence of a relatively high dose of exogenous TGF $\beta 3$ $(10 \mathrm{ng} / \mathrm{ml})$, alludes to the presence of additional factor(s) necessary for chondrogenic induction. Accordingly, we did not detect significant differences in the expression of TGF $\beta$ between PEMFed and non-PEMFed MSCs (Fig. 10) and moreover, TGF $\beta 1$ secretion was instead reduced in the PCM. By contrast, PEMF exposure of MSCs produced $\geq 2$-fold increases in the expressions of 
BMP2, BMP4, TSP2, and IL1ra that were further corroborated at the protein level using antibody arrays. BMP2 [56], BMP4 [57], and TSP2 [58-60] have been previously implicated in chondrogenic induction. Indeed, autocrine action of TSP-2 from umbilical cord MSCs has been shown to suppress hypertrophic phenotype development [60] and could possibly account for our observed suppression of MSC and chondrocyte hypertrophy in response to administration of PCM. BMP2 and BMP4, on the other hand, are implicated as chemotactic agents for MSCs [61] and could be responsible for the enhanced migratory effect we observed exerted by PCM on chondrocytes and MSCs. Finally, IL-1ra is the biological inhibitor of IL- $1 \beta$, associated with the severity of diseased cartilage degeneration [62] and has been demonstrated to reduce cartilage catabolism [63]. Nonetheless, the screening and confirmation of candidate factors participating in the chondrogenic enhancement observed with PCM is far from complete. In addition, the possible role of exosomal CD73-mediated adenosine activation of AKT, ERK, and AMPK signaling [14, 64] and the participation of exosomal microRNAs $[65,66]$ that have been previously implicated in regulating chondrocyte anabolic activity and cartilage degradation have yet to be examined. Further work will be required to fully decipher the paracrine mechanisms responsible for the enhanced anabolic and protective effect observed here in response to PCM administration, including the examination of the contribution of EVs to our reported PEMF-mediated secretome responses.

PEMF stimulation has been previously shown by others to exert anti-inflammatory effects by activating adenosine receptors [41, 44], while modulating intracellular calcium and activating the mechanotransduction FAK/Rho GTPases signaling pathways to induce MSC migration [67]. PEMF exposure was also shown to activate a calcium-mitochondrial axis stimulating mitochondrial respiration and promoting both mitochondriogenesis and myogenesis as well as reducing basal apoptosis and increasing telomere length via a process of adaptive magnetic mitohormesis [35]. Uniting these seemingly disparate responses is the finding that mechanical stimulation enhances mitochondrial ROS formation [30]. Accordingly, the generation of mitochondrial ROS has been shown to stimulate the secretome activity and is required for the development of the cellular and organismal adaptations against cellular inflammation [33]. It thus appears that mechanotransduction and mitochondrial respiration act upstream of secretome-mediated anti-inflammatory responses and appear to be modulated by magnetic fields as reported here. Subsequent studies will examine the mitochondrial contributions to the MSC secretome modulation induced by PEMF exposure.

We have previously demonstrated cell type-specific electromagnetic efficacy windows for PEMF-induced cytotoxicity of breast cancer cells [68], myogenic induction [35], or
MSC-based chondrogenic induction [34]. Here, we extend the biophysical criteria defining the electromagnetic efficacy window by showing that the culturing microenvironment could modulate magnetic sensitivity of MSCs and downstream secretome activity. 2D and 3D MSC culture platforms exhibited distinct sensitivities to PEMF stimulation; a predominance of cell-cell interactions in the 3D configuration gave rise to higher magnetic sensitivity with lower activation window. The optimum PEMF amplitude to produce PCM with enhanced biological efficacy from 2D and 3D MSC cultures was $3 \mathrm{mT}$ and $2 \mathrm{mT}$, respectively. MSCs grown on 2D TCP adopted fibroblastic morphologies and exhibited a predominance of cell-substrate interactions, whereas MSCs in 3D exhibited rounded morphologies and were largely dominated by cell-cell contacts. These distinct mechano-environmental scenarios likely elicited different mechanotransduction responses, which in turn converged with, or differently conditioned, the cellular response to magnetic field stimulation. For instance, cellular aggregation has been shown to trigger cadherin-related cell-cell interactions, which in itself augments MSC-dependent wound healing, myogenic, antiinflammatory, and angiogenic responses [27-29, 48]. It is thus possible that altering the cellular mechanical environment would influence MSC secretome composition and function as well as response and sensitivity to magnetic field exposure. Despite differences in PEMF sensitivities, similar levels of chondrogenic outcome and cartilage formation resulted from either 2D or 3D PCM administration (Figs. 3, 4, and 5). It remains to be resolved, however, whether any differences exist in the exact secretome profile of the PCM (or CCM) harvested from either platform.

\section{Conclusions}

We provide evidence that brief exposure to low amplitude PEMFs enhanced the ability of MSCs to produce and secrete paracrine factors capable of promoting cartilage regeneration as well as protecting against adverse inflammatory conditions. Furthermore, this report highlights the importance of optimizing PEMF exposure parameters for MSCs subjected to different culturing conditions. Collectively, our results indicate that PEMF stimulation could augment the production and release of the MSC paracrine repertoire for the ultimate enhancement of cartilage regeneration.

\section{Additional file}

Additional file 1: Table S1. A) Human PCR primer sequences. B)

Porcine primer sequence (DOCX $13 \mathrm{~kb})$

\section{Abbreviations}

ALP: Alkaline phosphatase; BMP2: Bone morphogenetic protein 2; BMP4: Bone morphogenetic protein 4; CAMP: Cyclic adenosine monophosphate; CM: Conditioned medium; CCM: Non-PEMFed CM; Col 
2: Type II Collagen; Col 1: Type I Collagen; Col 10: Type X Collagen; COX2: Cycloxigenase-2; ECM: Extracellular matrix; EVs: Extracellular vesicles; IGF: Insulin-like growth factor; IL-13: Interleukin-1 beta; IL-6: Interleukin 6; IL10: Interleukin 10; IL-1ra: Interleukin 1 receptor antagonist;

MSC: Mesenchymal stem cell; MMP13: Metalloproteinase 13; NOS: Nitric oxide synthase; PCM: PEMFed generated CM; PEMF: Pulsed electromagnetic field; PGE2: Prostaglandin E2; PCR: Polymerase chain reaction; sGAG: Sulfated glycosaminoglycan; Sox9: SRY-Box 9; TGF: Transforming growth factor; TRP: Transient receptor potential; TSP: Thrombospondin

\section{Acknowledgements}

The authors acknowledge Zac Goh (iHealthtech, National University of Singapore) for the graphical abstract shown in Fig. 1.

\section{Funding}

The study was supported by National Medical Research Council of Singapore (NMRC/CIRG /1403/2014) and the Lee Foundation of Singapore. Dinesh Parate was supported by NUS Research scholarship and NUS Department of Surgery Funds.

\section{Availability of data and materials}

The authors declare that the data supporting the findings of this study are available within the article and its supplementary information files.

\section{Authors' contributions}

DP conducted the bulk experimentation and analyzed the data as well as contributed to the writing of the manuscript. NDK and CC executed some of the experiments, participated in data acquisition and analysis. AF-O and EHL acquired funding and edited the manuscript. JHPH edited the manuscript. AF-O provided the PEMF device and expertise, conceptualized and designed the study, and participated in manuscript writing. ZY designed the study, interpreted the data, and wrote the manuscript. All authors read and approved the final manuscript.

\section{Ethics approval and consent to participate}

Not applicable.

\section{Consent for publication}

Not applicable.

\section{Competing interests}

AF-O is an inventor on patent WO 2019/17863 A1, System and Method for Applying Pulsed Electromagnetic Fields, and contributes to QuantumTx Pte Ltd., which elaborates on the use of similar magnetic fields for human use. All other authors declare that they have no competing interests.

\section{Author details}

'Department of Surgery, National University of Singapore, Singapore 119228, Singapore. ${ }^{2}$ Biolonic Currents Electromagnetic Pulsing Systems Laboratory, BICEPS, National University of Singapore, Singapore, Singapore. ${ }^{3}$ Department of Orthopaedic Surgery, Yong Loo Lin School of Medicine, National University of Singapore, NUHS Tower Block, Level 11, 1E Kent Ridge Road, Singapore 119288, Singapore. ${ }^{4}$ Tissue Engineering Program, Life Sciences Institute, National University of Singapore, DSO (Kent Ridge) Building, \#04-01, 27 Medical Drive, Singapore 117510, Singapore. Institute for Health Innovation \& Technology, iHealthtech, National University of Singapore, Singapore, Singapore.

\section{Received: 19 November 2019 Revised: 15 January 2020} Accepted: 20 January 2020 Published online: 03 February 2020

\section{References}

1. Marcacci M, Filardo G, Kon E. Treatment of cartilage lesions: what works and why? Injury. 2013;44(Suppl 1):S11-5.

2. Loeser RF, Goldring SR, Scanzello CR, Goldring MB. Osteoarthritis: a disease of the joint as an organ. Arthritis Rheum. 2012;64(6):1697-707.

3. Pittenger MF, Mackay AM, Beck SC, Jaiswal RK, Douglas R, Mosca JD, et al. Multilineage potential of adult human mesenchymal stem cells. Science. 1999;284(5411):143-7.

4. Toh WS, Brittberg M, Farr J, Foldager CB, Gomoll AH, Hui JH, et al. Cellular senescence in aging and osteoarthritis. Acta Orthop. 2016;87(sup363):6-14.
5. Somoza RA, Welter JF, Correa D, Caplan Al. Chondrogenic differentiation of mesenchymal stem cells: challenges and unfulfilled expectations. Tissue Eng Part B Rev. 2014;20(6):596-608.

6. Wu L, Leijten JC, Georgi N, Post JN, van Blitterswijk CA, Karperien M. Trophic effects of mesenchymal stem cells increase chondrocyte proliferation and matrix formation. Tissue Eng Part A. 2011;17(9-10):1425-36.

7. Levorson EJ, Santoro M, Kasper FK, Mikos AG. Direct and indirect co-culture of chondrocytes and mesenchymal stem cells for the generation of polymer/ extracellular matrix hybrid constructs. Acta Biomater. 2014;10(5):1824-35.

8. Maumus M, Jorgensen C, Noel D. Mesenchymal stem cells in regenerative medicine applied to rheumatic diseases: role of secretome and exosomes. Biochimie. 2013;95(12):2229-34.

9. Ha CW, Park YB, Kim SH, Lee HJ. Intra-articular mesenchymal stem cells in osteoarthritis of the knee: a systematic review of clinical outcomes and evidence of cartilage repair. Arthroscopy. 2019;35(1):277-88. e2

10. Kupcova SH. Proteomic techniques for characterisation of mesenchymal stem cell secretome. Biochimie. 2013;95(12):2196-211.

11. Van Linthout S, Stamm C, Schultheiss HP, Tschope C. Mesenchymal stem cells and inflammatory cardiomyopathy: cardiac homing and beyond. Cardiol Res Pract. 2011;2011:757154.

12. Oh JY, Lee RH, Yu JM, Ko JH, Lee HJ, Ko AY, et al. Intravenous mesenchymal stem cells prevented rejection of allogeneic corneal transplants by aborting the early inflammatory response. Mol Ther. 2012;20(11):2143-52.

13. Zhang S, Chu WC, Lai RC, Lim SK, Hui JH, Toh WS. Exosomes derived from human embryonic mesenchymal stem cells promote osteochondral regeneration. Osteoarthr Cartil. 2016;24(12):2135-40.

14. Zhang S, Chuah SJ, Lai RC, Hui JHP, Lim SK, Toh WS. MSC exosomes mediate cartilage repair by enhancing proliferation, attenuating apoptosis and modulating immune reactivity. Biomaterials. 2018;156:16-27.

15. Wang Y, Yu D, Liu Z, Zhou F, Dai J, Wu B, et al. Exosomes from embryonic mesenchymal stem cells alleviate osteoarthritis through balancing synthesis and degradation of cartilage extracellular matrix. Stem Cell Res Ther. 2017;8(1):189.

16. Cosenza S, Ruiz M, Toupet K, Jorgensen C, Noel D. Mesenchymal stem cells derived exosomes and microparticles protect cartilage and bone from degradation in osteoarthritis. Sci Rep. 2017;7(1):16214.

17. Murphy MB, Moncivais K, Caplan Al. Mesenchymal stem cells: environmentally responsive therapeutics for regenerative medicine. Exp Mol Med. 2013;45:e54.

18. Kusuma GD, Carthew J, Lim R, Frith JE. Effect of the microenvironment on mesenchymal stem cell paracrine signaling: opportunities to engineer the therapeutic effect. Stem Cells Dev. 2017;26(9):617-31.

19. Rodriguez TM, Saldias A, rigo M, Zamora JV, Perone MJ, Dewey RA. Effect of TGF-beta1 stimulation on the secretome of human adipose-derived Mesenchymal stromal cells. Stem Cells Transl Med. 2015;4(8):894-8.

20. Li C, Li G, Liu M, Zhou T, Zhou H. Paracrine effect of inflammatory cytokineactivated bone marrow mesenchymal stem cells and its role in osteoblast function. J Biosci Bioeng. 2016;121(2):213-9.

21. Teixeira FG, Panchalingam KM, Anjo SI, Manadas B, Pereira R, Sousa N, et al. Do hypoxia/normoxia culturing conditions change the neuroregulatory profile of Wharton jelly mesenchymal stem cell secretome? Stem Cell Res Ther. 2015;6:133.

22. Paquet J, Deschepper M, Moya A, Logeart-Avramoglou D, Boisson-Vidal C, Petite H. Oxygen tension regulates human mesenchymal stem cell paracrine functions. Stem Cells Transl Med. 2015;4(7):809-21.

23. Sullivan KE, Quinn KP, Tang KM, Georgakoudi I, Black LD 3rd. Extracellular matrix remodeling following myocardial infarction influences the therapeutic potential of mesenchymal stem cells. Stem Cell Res Ther. 2014;5(1):14.

24. Jose S, Hughbanks ML, Binder BY, Ingavle GC, Leach JK. Enhanced trophic factor secretion by mesenchymal stem/stromal cells with glycine-histidinelysine (GHK)-modified alginate hydrogels. Acta Biomater. 2014;10(5):1955-64.

25. Seib FP, Prewitz M, Werner C, Bornhauser M. Matrix elasticity regulates the secretory profile of human bone marrow-derived multipotent mesenchymal stromal cells (MSCs). Biochem Biophys Res Commun. 2009;389(4):663-7.

26. Yang H, Nguyen KT, Leong DT, Tan NS, Tay CY. Soft material approach to induce oxidative stress in mesenchymal stem cells for functional tissue repair. ACS Appl Mater Interfaces. 2016;8(40):26591-9.

27. Santos JM, Camoes SP, Filipe E, Cipriano M, Barcia RN, Filipe M, et al. Threedimensional spheroid cell culture of umbilical cord tissue-derived mesenchymal stromal cells leads to enhanced paracrine induction of wound healing. Stem Cell Res Ther. 2015;6:90.

28. Lee EJ, Park SJ, Kang SK, Kim GH, Kang HJ, Lee SW, et al. Spherical bullet formation via E-cadherin promotes therapeutic potency of mesenchymal 
stem cells derived from human umbilical cord blood for myocardial infarction. Mol Ther. 2012;20(7):1424-33.

29. Bartosh TJ, Ylostalo JH, Mohammadipoor A, Bazhanov N, Coble K, Claypool K, et al. Aggregation of human mesenchymal stromal cells (MSCs) into 3D spheroids enhances their antiinflammatory properties. Proc Natl Acad Sci U S A. 2010;107(31):13724-9.

30. Chatterjee S, Fisher AB. Mechanotransduction: forces, sensors, and redox signaling. Antioxid Redox Signal. 2014;20(6):868-71.

31. Kasper G, Dankert N, Tuischer J, Hoeft M, Gaber T, Glaeser JD, et al. Mesenchymal stem cells regulate angiogenesis according to their mechanical environment. Stem Cells. 2007;25(4):903-10.

32. Gardner OF, Fahy N, Alini M, Stoddart MJ. Differences in human mesenchymal stem cell secretomes during chondrogenic induction. Eur Cell Mater. 2016;31:221-35.

33. Scheele C, Nielsen S, Pedersen BK. ROS and myokines promote muscle adaptation to exercise. Trends Endocrinol Metab. 2009;20(3):95-9.

34. Parate D, Franco-Obregon A, Frohlich J, Beyer C, Abbas AA, Kamarul T, et al. Enhancement of mesenchymal stem cell chondrogenesis with short-term low intensity pulsed electromagnetic fields. Sci Rep. 2017:7(1):9421.

35. Yap JL, Tai YK, Fröhlich J, Fong CHH, Yin JN, Foo ZL, Ramanan S, Beyer C, Toh SJ, Casarosa M, Bharathy N, Kala MP, Egli M, Taneja, Lee CN, FrancoObregón A. Ambient and supplemental magnetic fields promote myogenesis via a TRPC1-mitochondrial axis: evidence of a magnetic mitohormetic mechanism. FASEB J. 2019;33(11):12853-12872. https://doi. org/10.1096/fj.201900057R.

36. Aaron RK, Wang S, Ciombor DM. Upregulation of basal TGFbeta1 levels by EMF coincident with chondrogenesis--implications for skeletal repair and tissue engineering. J Orthop Res. 2002;20(2):233-40.

37. Jansen $J \mathrm{H}$, van der Jagt OP, Punt BJ, Verhaar JA, van Leeuwen JP, Weinans $\mathrm{H}$, et al. Stimulation of osteogenic differentiation in human osteoprogenitor cells by pulsed electromagnetic fields: an in vitro study. BMC Musculoskelet Disord. 2010;11:188.

38. Amin HD, Brady MA, St-Pierre JP, Stevens MM, Overby DR, Ethier CR. Stimulation of chondrogenic differentiation of adult human bone marrowderived stromal cells by a moderate-strength static magnetic field. Tissue Eng Part A. 2014;20(11-12):1612-20.

39. Kwon $\mathrm{HJ}$, Lee GS, Chun $\mathrm{H}$. Electrical stimulation drives chondrogenesis of mesenchymal stem cells in the absence of exogenous growth factors. Sci Rep. 2016;6:39302.

40. Varani K, Padovan M, Vincenzi F, Targa M, Trotta F, Govoni M, et al. A2A and A3 adenosine receptor expression in rheumatoid arthritis: upregulation, inverse correlation with disease activity score and suppression of inflammatory cytokine and metalloproteinase release. Arthritis Res Ther. 2011;13(6):R197.

41. Varani K, Vincenzi F, Ravani A, Pasquini S, Merighi S, Gessi S, et al. Adenosine receptors as a biological pathway for the anti-inflammatory and beneficial effects of low frequency low energy pulsed electromagnetic fields. Mediat Inflamm. 2017:2017:2740963.

42. De Mattei M, Varani K, Masieri FF, Pellati A, Ongaro A, Fini M, et al. Adenosine analogs and electromagnetic fields inhibit prostaglandin E2 release in bovine synovial fibroblasts. Osteoarthr Cartil. 2009;17(2):252-62.

43. Ongaro A, Pellati A, Setti S, Masieri FF, Aquila G, Fini M, et al. Electromagnetic fields counteract IL-1 beta activity during chondrogenesis of bovine mesenchymal stem cells. J Tissue Eng Regen Med. 2015;9(12):E229-38.

44. Ongaro A, Varani K, Masieri FF, Pellati A, Massari L, Cadossi R, et al. Electromagnetic fields (EMFs) and adenosine receptors modulate prostaglandin E (2) and cytokine release in human osteoarthritic synovial fibroblasts. J Cell Physiol. 2012;227(6):2461-9.

45. Raghothaman D, Leong MF, Lim TC, Toh JK, Wan AC, Yang Z, et al. Engineering cell matrix interactions in assembled polyelectrolyte fiber hydrogels for mesenchymal stem cell chondrogenesis. Biomaterials. 2014; 35(9):2607-16.

46. Yang Z, Wu Y, Li C, Zhang T, Zou Y, Hui JH, et al. Improved mesenchymal stem cells attachment and in vitro cartilage tissue formation on chitosanmodified poly(L-lactide-co-epsilon-caprolactone) scaffold. Tissue Eng Part A. 2012;18(3-4):242-51

47. Yin L, Wu Y, Yang Z, Denslin V, Ren X, Tee CA, et al. Characterization and application of size-sorted zonal chondrocytes for articular cartilage regeneration. Biomaterials. 2018;165:66-78.

48. Qazi TH, Mooney DJ, Duda GN, Geissler S. Biomaterials that promote cellcell interactions enhance the paracrine function of MSCs. Biomaterials. 2017; 140:103-14.
49. Goldring MB, Tsuchimochi K, ljiri K. The control of chondrogenesis. J Cell Biochem. 2006;97(1):33-44.

50. Li Z, Kupcsik L, Yao SJ, Alini M, Stoddart MJ. Mechanical load modulates chondrogenesis of human mesenchymal stem cells through the TGF-beta pathway. J Cell Mol Med. 2010;14(6A):1338-46.

51. Zhu Y, Wang Y, Zhao B, Niu X, Hu B, Li Q, et al. Comparison of exosomes secreted by induced pluripotent stem cell-derived mesenchymal stem cells and synovial membrane-derived mesenchymal stem cells for the treatment of osteoarthritis. Stem Cell Res Ther. 2017:8(1):64.

52. Vonk LA, van Dooremalen SFJ, Liv N, Klumperman J, Coffer PJ, Saris DBF, et al. Mesenchymal stromal/stem cell-derived extracellular vesicles promote human cartilage regeneration in vitro. Theranostics. 2018;8(4):906-20.

53. Glyn-Jones S, Palmer AJ, Agricola R, Price AJ, Vincent TL, Weinans H, et al. Osteoarthritis. Lancet. 2015;386(9991):376-87.

54. Hunziker EB, Rosenberg LC. Repair of partial-thickness defects in articular cartilage: cell recruitment from the synovial membrane. J Bone Joint Surg Am. 1996;78(5):721-33.

55. Zhang W, Chen J, Tao J, Jiang Y, Hu C, Huang L, et al. The use of type 1 collagen scaffold containing stromal cell-derived factor-1 to create a matrix environment conducive to partial-thickness cartilage defects repair. Biomaterials. 2013;34(3):713-23.

56. Schmitt B, Ringe J, Haupl T, Notter M, Manz R, Burmester GR, et al. BMP2 initiates chondrogenic lineage development of adult human mesenchymal stem cells in high-density culture. Differentiation. 2003;71(9-10):567-77.

57. Jiang Y, Chen LK, Zhu DC, Zhang GR, Guo C, Qi YY, et al. The inductive effect of bone morphogenetic protein-4 on chondral-lineage differentiation and in situ cartilage repair. Tissue Eng Part A. 2010;16(5):1621-32.

58. Taylor DK, Meganck JA, Terkhorn S, Rajani R, Naik A, O'Keefe RJ, et al. Thrombospondin-2 influences the proportion of cartilage and bone during fracture healing. J Bone Miner Res. 2009;24(6):1043-54.

59. Jeong SY, Kim DH, Ha J, Jin HJ, Kwon SJ, Chang JW, et al. Thrombospondin2 secreted by human umbilical cord blood-derived mesenchymal stem cells promotes chondrogenic differentiation. Stem Cells. 2013;31(10):2136-48.

60. Jeong SY, Ha J, Lee M, Jin HJ, Kim DH, Choi SJ, et al. Autocrine action of thrombospondin-2 determines the chondrogenic differentiation potential and suppresses hypertrophic maturation of human umbilical cord bloodderived mesenchymal stem cells. Stem Cells. 2015:33(11):3291-303.

61. Fiedler J, Roderer G, Gunther KP, Brenner RE. BMP-2, BMP-4, and PDGF-bb stimulate chemotactic migration of primary human mesenchymal progenitor cells. J Cell Biochem. 2002;87(3):305-12.

62. Lamacchia C, Rodriguez E, Palmer G, Vigne S, Martin P, Talabot-Ayer D, et al. Articular inflammation is controlled by myeloid cell-derived interleukin 1 receptor antagonist during the acute phase of arthritis in mice. Ann Rheum Dis. 2012;71(2):281-7.

63. Elsaid KA, Zhang L, Shaman Z, Patel C, Schmidt TA, Jay GD. The impact of early intra-articular administration of interleukin-1 receptor antagonist on lubricin metabolism and cartilage degeneration in an anterior cruciate ligament transection model. Osteoarthr Cartil. 2015;23(1):114-21.

64. Zhang S, Teo KYW, Chuah SJ, Lai RC, Lim SK, Toh WS. MSC exosomes alleviate temporomandibular joint osteoarthritis by attenuating inflammation and restoring matrix homeostasis. Biomaterials. 2019;200:35-47.

65. Wu J, Kuang L, Chen C, Yang J, Zeng WN, Li T, et al. miR-100-5p-abundant exosomes derived from infrapatellar fat pad MSCs protect articular cartilage and ameliorate gait abnormalities via inhibition of mTOR in osteoarthritis. Biomaterials. 2019:206:87-100.

66. Mao G, Hu S, Zhang Z, Wu P, Zhao X, Lin R, et al. Exosomal miR-95-5p regulates chondrogenesis and cartilage degradation via histone deacetylase 2/8. J Cell Mol Med. 2018;22(11):5354-66.

67. Zhang $Y$, Yan J, Xu H, Yang Y, Li W, Wu H, et al. Extremely low frequency electromagnetic fields promote mesenchymal stem cell migration by increasing intracellular $\mathrm{Ca}(2+)$ and activating the FAK/Rho GTPases signaling pathways in vitro. Stem Cell Res Ther. 2018;9(1):143.

68. Crocetti S, Beyer C, Schade G, Egli M, Frohlich J, Franco-Obregon A. Low intensity and frequency pulsed electromagnetic fields selectively impair breast cancer cell viability. PLoS One. 2013;8(9):e72944.

\section{Publisher's Note}

Springer Nature remains neutral with regard to jurisdictional claims in published maps and institutional affiliations. 